ARTICLE

\title{
Pooled CRISPRi screening of the cyanobacterium Synechocystis sp PCC 6803 for enhanced industrial phenotypes
}

Lun Yao 1,2,3, Kiyan Shabestary (10) 1,2,3, Sara M. Björk (iD 1,2, Johannes Asplund-Samuelsson (iD 1,2, Haakan N. Joensson (D) 1,2, Michael Jahn (1) ${ }^{1,2} \&$ Elton P. Hudson (1) ${ }^{1,2 \times}$

Cyanobacteria are model organisms for photosynthesis and are attractive for biotechnology applications. To aid investigation of genotype-phenotype relationships in cyanobacteria, we develop an inducible CRISPRi gene repression library in Synechocystis sp. PCC 6803, where we aim to target all genes for repression. We track the growth of all library members in multiple conditions and estimate gene fitness. The library reveals several clones with increased growth rates, and these have a common upregulation of genes related to cyclic electron flow. We challenge the library with $0.1 \mathrm{M} \mathrm{L-lactate}$ and find that repression of peroxiredoxin bcp2 increases growth rate by $49 \%$. Transforming the library into an L-lactatesecreting Synechocystis strain and sorting top lactate producers enriches clones with sgRNAs targeting nutrient assimilation, central carbon metabolism, and cyclic electron flow. In many examples, productivity can be enhanced by repression of essential genes, which are difficult to access by transposon insertion.

\footnotetext{
${ }^{1}$ Science for Life Laboratory, KTH - Royal Institute of Technology, SE-171 21 Stockholm, Sweden. ${ }^{2}$ Department of Protein Science, KTH - Royal Institute of Technology, SE-106 91 Stockholm, Sweden. ${ }^{3}$ These authors contributed equally: Lun Yao, Kiyan Shabestary. ${ }^{凶}$ email: paul.hudson@scilifelab.se
} 
C yanobacteria are model organisms for photosynthetic electron flow, photorespiration, and the circadian clock ${ }^{1-3}$. In addition to their massive ecological importance, biotechnological applications of cyanobacteria have been proposed, such as microbial cell factories, where metabolism is engineered to synthesize chemicals from $\mathrm{CO}_{2}$ using energy derived from light ${ }^{4-6}$. This widespread interest in cyanobacteria brings a need for a system-wide analysis of gene essentiality, as well as tools for elucidating gene function. Even in model cyanobacteria such as Synechocystis, approximately $45 \%$ of genes have no assigned function (Cyanobase). Historically, the transposon mutagenesis library has been used to identify loss-of-function or gain-of-function mutants in bacteria. Variants of transposon mutagenesis tag the transposon insertion site with a barcode, which enables tracking and quantification of mutant growth via next-generation sequencing (NGS). Rapid screening of libraries in multiple growth conditions is one avenue to elucidating gene function ${ }^{7}$. A transposon library in the cyanobacterium Synechococcus elongatus PCC 7942 (hereafter Synechococcus PCC 7942) was used to map essential genes in both constant light and diurnal growth (12 h light, $12 \mathrm{~h}$ dark $)^{8,9}$.

An alternative to transposon mutant libraries are pooled CRISPRi libraries for targeted gene repression, where unique single-guide RNA (sgRNA) genes are pooled and transformed into the strain of interest. Since the protospacer region of sgRNAs is small enough $(\sim 20 \mathrm{nt})$ to be sequenced by NGS, it can serve as a barcode and allows monitoring of the abundance of each clone in the library. Important for phenotyping, inducible CRISPRi allows modulation of essential genes. CRISPRi libraries have been applied to screen gene essentiality and diverse phenotypes in various model bacteria, including morphology, solvent tolerance, and phage resistance ${ }^{10-12}$. Inducible CRISPRi has been developed for several cyanobacteria strains $^{13,14}$, but pooled sgRNA libraries have not been exploited.

Here we report the construction and use of an inducible CRISPRi library of 10498 clones for the model cyanobacterium Synechocystis sp. PCC 6803 (hereafter Synechocystis). Each clone expresses dCas 9 and an sgRNA that targets a protein-coding open reading frame (ORF) or a non-coding RNA (ncRNA). We track the composition of the library during growth in multiple conditions, including different light regimes and in the presence of the organic acid L-lactate. We also screen the library for enhanced Llactate production using droplet microfluidics (Fig. 1). In addition to providing fitness scores for almost all ORFs and ncRNAs in Synechocystis, our results give insights into how to engineer cyanobacteria metabolism for industrial use. We show that there are multiple gene knockdowns that increase the growth rate of Synechocystis, though these genes regulate similar processes in photosynthesis. We find far fewer diurnal-specific fitness genes in Synechocystis when compared to previously published data from Synechococcus PCC 7942. Further, we provide support for previous computational predictions that alteration of the ATP/ NADPH balance can improve bioproduction in cyanobacteria. By screening both growth and productivity, this platform can yield mutants that trade biomass formation for increased productivity of a target compound. While we have focused on biotechnological traits of interest here, the CRISPRi library can be used to explore the connection between growth and robustness in many conditions, all while allowing access to modulation of essential genes. The data for all competition experiments performed with the sgRNA library can be accessed through an interactive web application [https://m-jahn.shinyapps.io/ShinyLib/].

\section{Results}

Gene fitness during photoautotrophic growth. We designed a CRISPRi library for repression of each annotated gene in Synechocystis (3546 ORFs, 1871 ncRNAs) ${ }^{15}$. We designed two sgRNAs for each of 3526 ORFs and 1555 ncRNAs, and one sgRNA for 20 ORFs and 316 ncRNAs, totalling 10498 sgRNAs (Methods, Supplementary Data 1, Supplementary Data 2). The resulting 10498 sgRNA sequences were synthesized, pooled, and cloned into a genomic integration vector, and the pooled sgRNAs were transformed into a Synechocystis strain containing a genomeintegrated, anhydrotetracycline (aTc)-inducible dCas9 cassette. The resulting Synechocystis clones were pooled and constituted the CRISPRi library. Deep sequencing of the sgRNA region of the library confirmed that all of the designed 10498 sgRNAs were present.

We cultivated the library in light-limited turbidostats under two constant-light conditions, $100 \mu \mathrm{mol}$ photons $\mathrm{m}^{-2} \mathrm{~s}^{-1}$ (L100) and $300 \mu \mathrm{mol}$ photons $\mathrm{m}^{-2} \mathrm{~s}^{-1}$ (L300) and a light-dark diurnal condition (LD, sinusoidal illumination up to $300 \mu \mathrm{mol}$ photons $\mathrm{m}^{-2} \mathrm{~s}^{-1}$ over a $12 \mathrm{~h}$ period followed by $12 \mathrm{~h}$ darkness), each with supplemented $1 \% \mathrm{CO}_{2}$ and in four replicates. Samples for NGS were taken periodically over 32 cell generations (L100 and LD cultivations were 32 days, $\mu \sim 0.03 \mathrm{~h}^{-1}$; L300 cultivations were 16 days, $\mu \sim 0.07 \mathrm{~h}^{-1}$; Supplementary Data 3$)$. For each sampling point, the abundance of the 7072 sgRNAs targeting ORFs were quantified and averaged across the four replicates. The sgRNAs were grouped into five clusters based on their rate of washout (depletion) from the turbidostat (Fig. 2a). In total, 1998 sgRNAs $(28.3 \%)$ had a significant depletion in at least one condition, indicating these target genes have some contribution to cell fitness (Fig. 2b). Cluster 1 (241 sgRNAs) contained sgRNAs that were depleted in induced and uninduced cultivations, revealing weak background leakage of dCas9 and particular sensitivity to changes in abundance of these genes. Cluster 2 (378 sgRNAs) was enriched in sgRNAs that were quickly depleted in all growth conditions. Cluster 3 (767 sgRNAs) contained sgRNAs that were more rapidly depleted in L300 than L100. Cluster 4 (612 sgRNAs) contained sgRNAs that were depleted slowly, and cluster 5 (5074 sgRNAs) contained sgRNAs that were not depleted at all. Cluster 5 combines two very similar clusters differing only in the temporary enrichment of a subset of sgRNAs, most likely due to technical variation (L300, 1 and $2 \mathrm{~d}$ time-points). A geneontology (GO term) enrichment analysis showed that clusters 1 and 2 were highly enriched for GO terms related to core cellular processes such as photosynthesis, carbon fixation, and translation (Fig. 2c). Cyanobacteria invest most of their resources into synthesizing proteins from these groups ${ }^{16,17}$. Interestingly, nearly all sgRNAs with a low fitness score (i.e., they are depleted from the library) targeted genes whose expression were found by Jahn et al. to be regulated with growth rate (Supplementary Fig. 1, protein abundance based on mass spectrometry measurements) ${ }^{16}$. Cluster 3 was enriched for a more diverse set of GO terms (e.g., secondary metabolites, cell membrane) and cluster 4 was enriched in only one GO term (nucleotide metabolism).

Fitness scores for genes in central carbon metabolism can be used to refine flux predictions of core metabolic models. Here, data revealed instances of both rigidity and plasticity in Synechocystis central carbon metabolism. For example, the production of pyruvate in Synechocystis has been predicted to be mainly through decarboxylation of oxaloacetate by NADPdependent malic enzyme, not dephosphorylation of phosphoenolpyruvate by pyruvate kinase ${ }^{18}$. However, malic enzyme ( $m e$, slr0721) and pyruvate kinase ( $p y k 2$, sll1275) both have low fitness scores (Supplementary Fig. 2). This suggests that one route to pyruvate cannot fully substitute for the loss of the other, though interpretation of $p y k 2$ fitness is complicated by its position in an operon (see Discussion). Within the Calvin cycle, two energetically equivalent routes for synthesis of fructose-6-phosphate have been considered: through class-I fructose-bisphosphate aldolase $(f d a, s l r 0943)$ or through transaldolase $(\operatorname{talB}, \operatorname{slr1793})^{19}$. Neither 

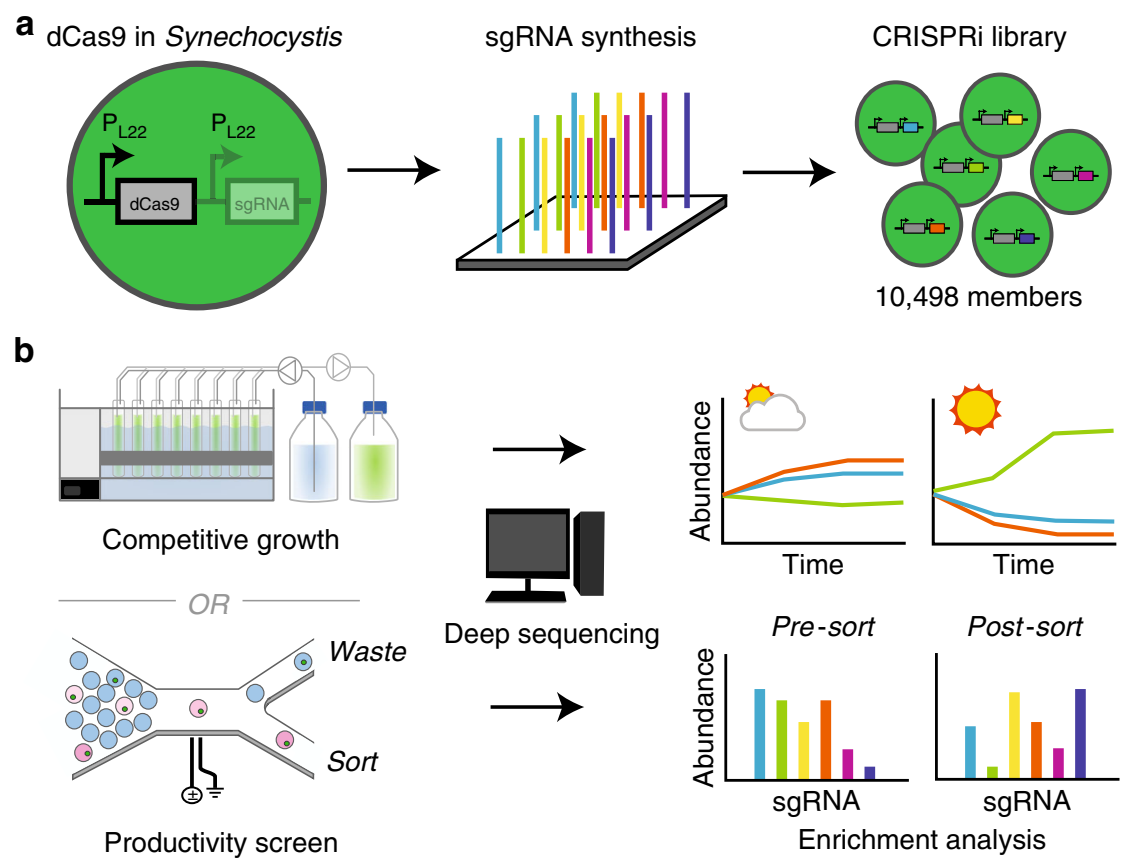

Fig. 1 Workflow for CRISPRi screening in Synechocystis. a Creating the CRISPRi library. An inducible promoter $\mathrm{P}_{\mathrm{L} 22}$ is used in Synechocystis. $\mathbf{b}$ The fitnesS or productivity of each library member is assessed by counting mutants in a bioreactor cultivation or in a sorted population using next-generation sequencing.

of these genes had a low fitness score, though the class-II FBP/ SBP aldolase gene ( $c b b A$, sllo018), which is needed to synthesize sedoheptulose bisphosphate for both proposed routes, did have a low fitness score. While these results do not definitively show which route is favored under photoautotrophic conditions, they suggest that flux can operate through either route if the other is perturbed. This flexibility is in contrast to Synechococcus PCC 7942, where a transposon insertion library showed that the sole FBP aldolase gene was essential for photoautotrophic growth, and transaldolase was not ${ }^{8}$.

We next compared fitness scores for sgRNAs in each growth condition (see Methods for calculation). Fitness scores were generally lower in the L300 condition than in L100, even when normalized to number of cell generations (Fig. 3a). One explanation could be a higher protein turnover rate in the higher growth condition. Lower fitness scores at L300 could also be expected for genes mediating high light or redox stress acclimation. In order to find genes important for specific light conditions, we calculated gene fitness as the mean fitness of two sgRNAs, and selected only genes where both sgRNAs were present in the same cluster. We found 38 genes that were beneficial for growth in L300 but neutral in L100, according to a difference in gene fitness $\geq 3$ (Fig. 3b). This set was almost exclusively in cluster 3 and 4 and included genes related to DNA repair, proteome and redox homeostasis, and subunits of PSI and the NADPH dehydrogenase NDH-1 (Fig. 3c). Energy dissipation and photo-stress response are thus particularly important for growth at high light. There were 22 genes with differential fitness between L100 and LD, and only 5 between L300 and LD (Supplementary Fig. 3 and 4). The extensive overlap between L300 and LD suggests that the LD condition may involve light stress, likely at dawn. The five genes with different fitness scores between $\mathrm{L} 300$ and $\mathrm{LD}$ include two $\mathrm{CO}_{2}$ hydration genes ( $n d h 3$, slr1302). The dearth of genes with LD-specific fitness contributions is in contrast to Synechococcus PCC 7942, where more than 100 genes were found with a transposon library to be important for LD growth ${ }^{9}$. This discrepancy could be attributed to the
CRISPRi library comprising partial repressions and not gene knockouts. Furthermore, Synechococcus has a more pronounced day-night rhythm than Synechocystis, with a higher fraction of oscillatory transcripts and larger amplitudes ${ }^{20}$.

Mutants with a growth advantage. Quantification of the library population over time in a turbidostat allows calculation of the maximum specific growth rate $\mu$ of each mutant (Methods). Growth-rate estimates allow for more intuitive interpretation of mutant fitness and highlights that library clones are repression mutants, not total knockouts. For example, repression of Calvin cycle genes often caused significant reduction of $\mu$ (e.g., prk $-40 \%, c b b A-95 \%$ ), while repression of most photorespirationrelated genes did not (e.g., glcD1 $-10 \%$, glcD2 -10\%; Supplementary Fig. 2). Many clones showed higher growth rates than the population average, notably those targeting pmgA (mean increase for L100 and L300 + 17\%) and slr1916 (mean increase + 13\%). The pmgA and slr1916 repression clones also showed higher growth rates in the diurnal condition (Fig. 4a). PmgA is a regulator involved in the high-light response in Synechocystis and a pmgA knockout mutant has an inability to reduce PSI content in high light, resulting in more efficient photosynthesis ${ }^{21}$. A slr1916 mutant was previously identified from a small Synechocystis transposon library on the basis of altered fluorescence kinetics and also shows a higher PSI content at high light ${ }^{22}$. Notably, other mutants isolated in the study of Ozaki et al. were not enriched in our library, indicating that altered PSI/PSII alone does not ensure faster growth ${ }^{22}$. Slr1916 was annotated based on homology as $m e n H$, an esterase in the phylloquinone pathway. However, repression of the 8 other phylloquinone pathway genes resulted in strong growth defects, suggesting slr1916 is not a key enzyme in this pathway (Supplementary Fig. 5). Furthermore, slr1916 is localized to the plasma membrane ${ }^{23}$, though its proposed substrates are soluble. Three additional sgRNA clones showed slight but significant increases in growth rate in the turbidostat data $(+5 \%)$ : sll1969, an annotated triacylglycerol 


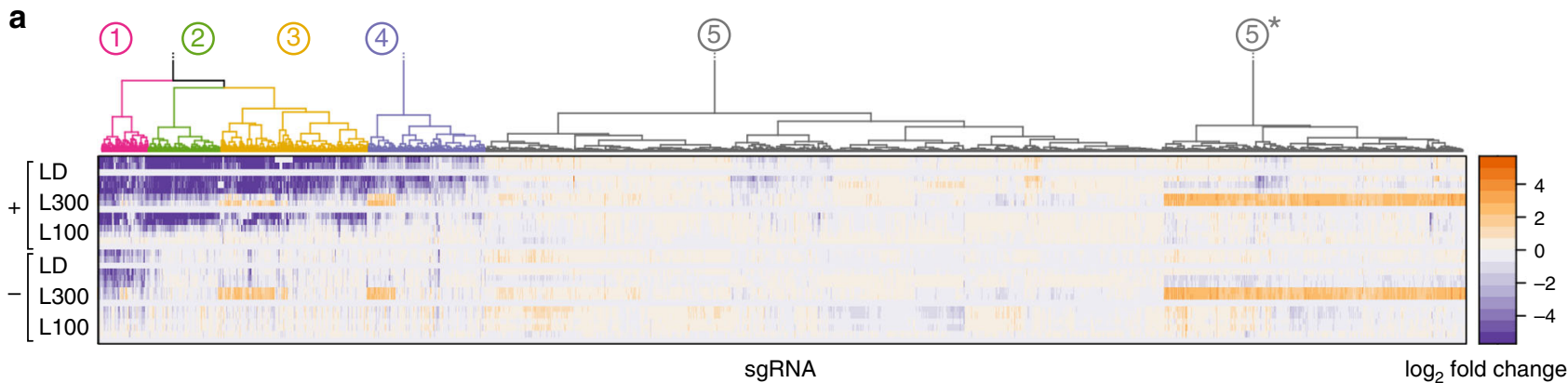

b
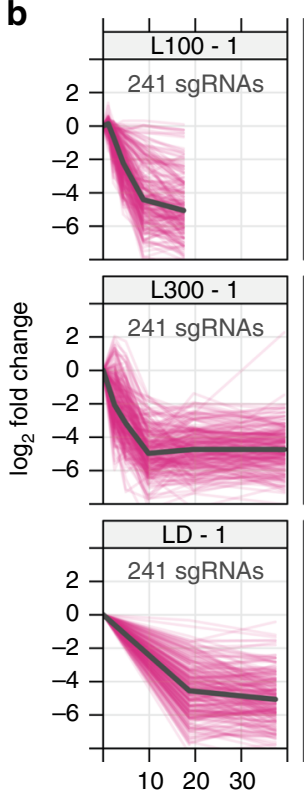

$10 \quad 20 \quad 30$
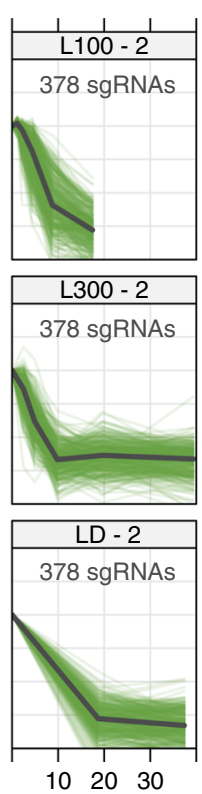
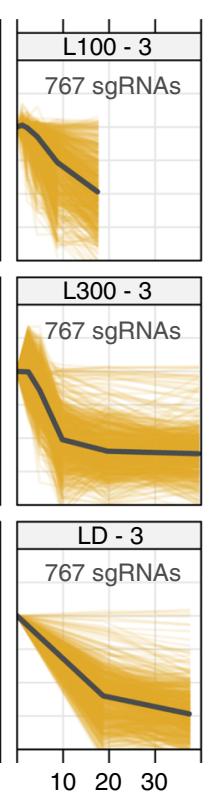

Generations
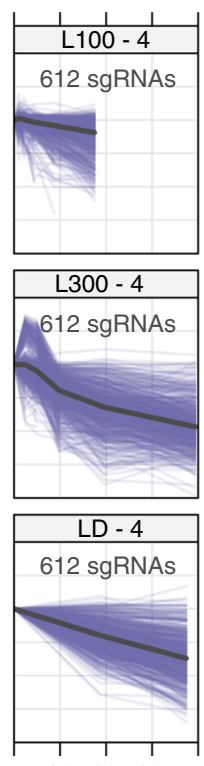

$10 \quad 20 \quad 30$
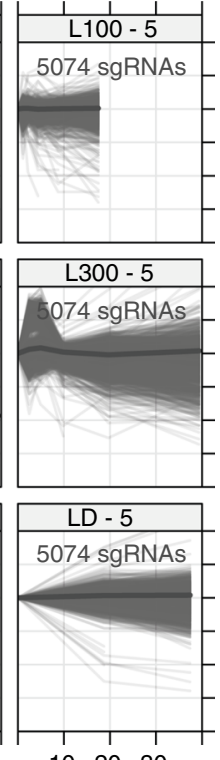

$10 \quad 20 \quad 30$
C

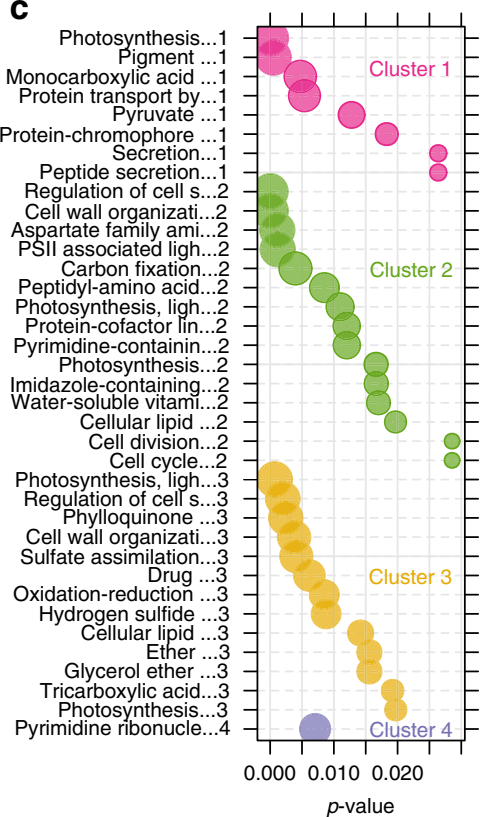

Fig. 2 Dynamics of CRISPRi library during photoautotrophic growth. a Clustered sgRNAs by similarity of $\log _{2}$ fold-change over time. Rows represent time-points sampled after induction. (L100-light with $100 \mu \mathrm{mol} \mathrm{m}-2 \mathrm{~s}^{-1}$, L300-light with $300 \mu \mathrm{mol} \mathrm{m} \mathrm{m}^{-2} \mathrm{~s}^{-1}$, LD-light-dark cycle). All log 2 fold-change values were calculated from averages of four replicate cultivations. Symbols: plus, induced, minus, non-induced, star, cluster 5 is a combination of two unchanged clusters. $\mathbf{b} \log _{2}$ fold-change of individual sgRNAs over the course of each cultivation. Experiment run time was normalized to number of cell generations estimated from population growth rate. c Enriched gene-ontology (GO) terms for the four clusters (1-4) showing sgRNA depletion; $p$-valueFisher's exact test with elimination (see Methods). Source data are provided as a Source Data file.

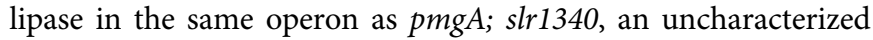
gene encoding a predicted acetyl-transferase, and ssl2982, encoding the non-essential $\omega$ subunit of RNA polymerase ${ }^{24}$.

In an attempt to uncover common regulations among these mutants, we reconstructed axenic knockdown strains. The repression clones of $p m g A$, slr1916, sll1969, and ssl2982 each had a higher maximum growth rate than the control strain, as measured in the exponential phase of batch cultivation, while the growth rate of the $\operatorname{slr} 1340$ repression clone was not higher than the control strain (Supplementary Fig. 6). We next collected transcriptomics of strains after CRISPRi induction. A pmgA clone was not included as microarray data from a $p m g A$ knockout was reported previously ${ }^{25}$. From the RNA-Seq data, slr1916 and ssl2982 mutants had 143 and 248 differentially expressed genes compared to a control strain (sgRNA-NT0, with no target site in Synechocystis genome) (Fig. 4b). A weak transcriptomic response of $\operatorname{sir} 1340$ and sll1969 mutants could be due to a lower repression efficiency in these clones ( $\log _{2}$ FC of target genes was -2.57 and -1.67 , for $s l r 1340$ and sll1969, respectively, compared to -5.37 and -4.85 for slr1916 and ssl2982). Clustering genes based on similarity of expression changes revealed that the same set of genes was affected for all mutants, but with stronger effects in slr1916 and ssl2982 (Fig. 4c). The high-light responsive transcription factor $\mathrm{RpaB}$ (slr0947, regulator of phycobilisome association B) was upregulated in multiple mutants. The extensive regulon of $\mathrm{RpaB}$ includes likely repressor activity of linear electron transport at high light (e.g., subunits of PSII and Cyt- $\mathrm{b}_{6} \mathrm{f}$ ), and activation activity of photoprotection and cyclic electron flow (e.g., ftsH and ssr2016) ${ }^{26}$. Other common upregulated genes are involved in electron transport ( $f e d 7)$, and include components of the NDH-1 complex (ndhD1, ndhD2) and carotenoid biosynthesis (crtQ and $c r t Z$ ) (Fig. 4d). Cyanobacterial NDH-1 complexes participate in cyclic electron flow around PSI, respiratory electron flow, and $\mathrm{CO}_{2}$ uptake ${ }^{27}$. Genes that were downregulated in all four mutants were sll1851, a small nonannotated gene, and $\operatorname{chlN}(\operatorname{slr} 0750)$, a subunit of the lightindependent operative protochlorophyllide oxidoreductase (LIPOR).

Gene fitness in the presence of L-lactate. The CRISPRi library is also beneficial for finding stress tolerance phenotypes, which are generally difficult to engineer rationally. The commodity chemical L-lactate has been produced in several cyanobacteria but still 

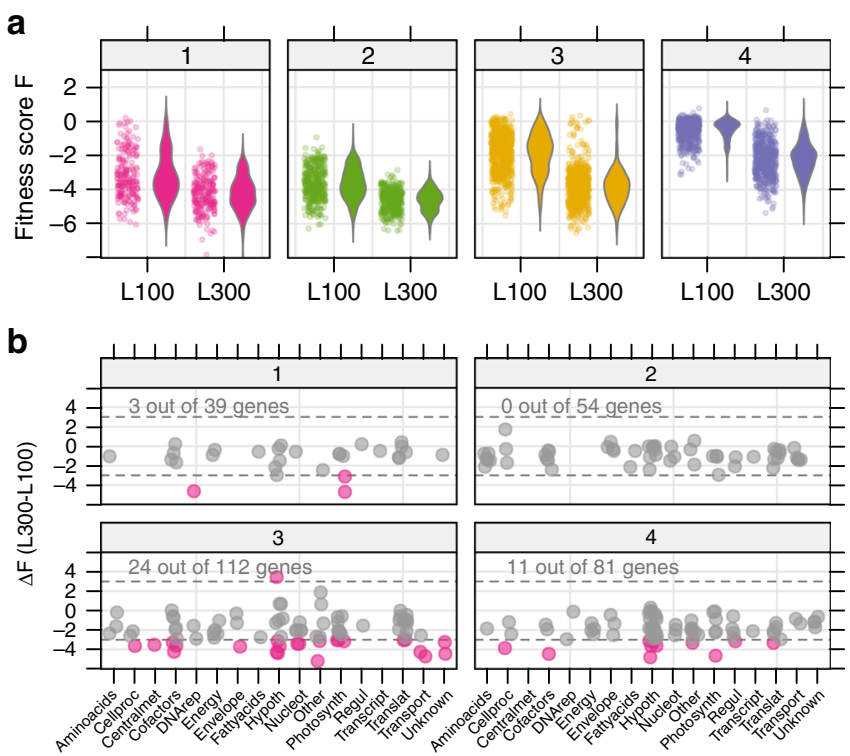

C

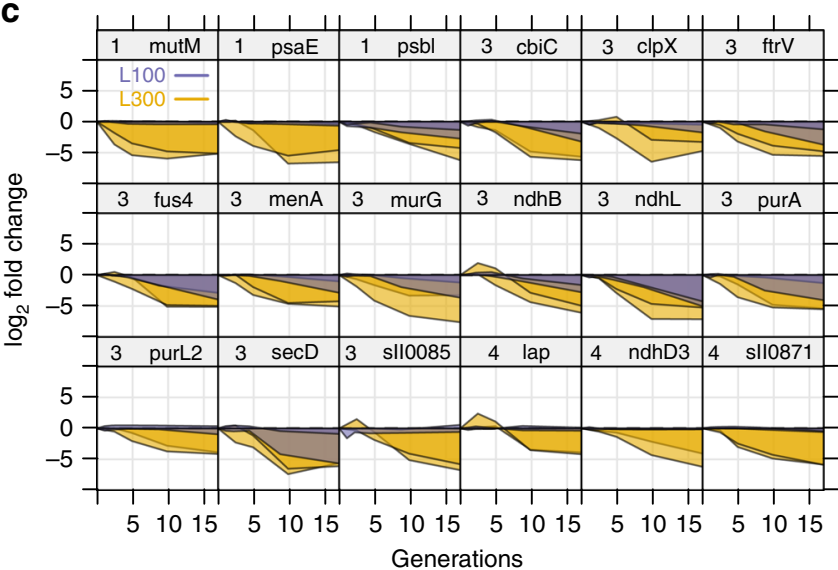

Fig. 3 Genes with condition-dependent fitness in two light conditions. a Distribution of fitness score $F$ for all sgRNAs in clusters 1-4 (color code as in Fig. 2). Fitness score indicates the degree of enrichment (positive) or depletion (negative) of an sgRNA, normalized to cell generations.

Comparison between two light conditions (L100-light with $100 \mu \mathrm{mol} \mathrm{m} \mathrm{m}^{-2}$ $\mathrm{s}^{-1}$ and L300-light with $300 \mu \mathrm{mol} \mathrm{m}^{-2} \mathrm{~s}^{-1}$ ) shows sgRNAs are on average more rapidly depleted under $\mathrm{L} 300$ independent of number of cell generations. $\mathbf{b}$ Difference in fitness $(\Delta F)$ between $\mathrm{L} 100$ and L300, for genes with both sgRNAs in the same cluster. Differentially depleted/enriched sgRNAs indicated in red, threshold: $3 \leq \Delta \mathrm{F} \leq-3$. c Time courses of both sgRNAs for a selection of 18 genes with high $\Delta F$ in L100 (blue) and L300 (yellow). Source data are provided as a Source Data file.

at relatively low titers (up to $15 \mathrm{mM}$ ) 28 . The tolerance of Synechocystis to L-lactate is $\sim 0.1 \mathrm{M}(9 \mathrm{~g} / \mathrm{L})$, at which specific growth rate is reduced by $50 \%$ (L100 condition, Supplementary Data 3). To identify mutants with increased tolerance to L-lactate, we cultivated the library in turbidostats with added $0.1 \mathrm{M}$ sodium Llactate (pH-adjusted) and sampled periodically for NGS $(0,16$, and $32 \mathrm{~d}$ ). We found 75 sgRNAs that were enriched during the Llactate cultivation, but not in a $\mathrm{NaCl}$ control cultivation (Fig. 5a). Eight genes were enriched with both sgRNAs (Fig. 5b). Curiously, 19 of the enriched sgRNAs (24\%) targeted genes in amino-acid metabolism and protein biosynthesis, including multiple aminoacid tRNA synthetases (argS, aspS, asnS, glyS, gltX, metS) (Supplementary Fig. 7). Many of these clones had growth defects in the absence of L-lactate $(\mathrm{NaCl}$ control, grey area in
Supplementary Fig. 6), which supports the general phenomenon that slow-growing microbes are more stress tolerant ${ }^{29}$.

We reconstructed nine of the repression clones to confirm growth improvement in batch cultures with added L-lactate (Fig. 5c, d, Supplementary Fig. 8). Of the nine clones, the sll1712 and $b c p 2$ mutants had significantly higher growth rates than the control strain (Fig. $5 c$ ). The $b c p 2$ mutant (bacterioferritin comigratory protein, a peroxiredoxin) had the largest improvement, a $49 \%$ increase in $\mu(p=0.006$, student's $t$-test). We tested Llactate consumption of all mutants and found that none consumed L-lactate over a 48 -hour period. The mechanism for increased tolerance in the $b c p 2$ mutant is not known. However, thioredoxins can mediate direct reduction of cysteines on transcription factors, including the master $\mathrm{RpaB}$ regulator in Synechocystis ${ }^{30}$. The absorbance spectra of all L-lactate-tolerant mutants showed increased chlorophyll $\mathrm{A}$ and phycocyanin absorption in the presence of L-lactate (Fig. 5e, f). Relative concentration of carotenoids, pigments related to the light stress response, was reduced in L-lactate tolerant mutants.

We could also identify clones with growth negatively affected by L-lactate but not by $\mathrm{NaCl}$ (Supplementary Fig. 9). These targeted genes could thus be candidates for overexpression to improve tolerance. Most prominent were an antibiotic resistance gene (zam, sll1910), two nucleases, the protease clpX (sll0535), cytochrome M (cytM, sll1245) that may act to dissipate excess electrons $^{31}$, and $\operatorname{sepF}$ (slr2073), an inhibitor of cell division.

Screening the CRISPRi library for L-lactate productivity. The CRISPRi library can be linked to screens other than growth, and we next sought to find mutant clones that had increased productivity of L-lactate. The sgRNA pool and inducible dCas9 cassette were cloned into a Synechocystis strain containing lactate dehydrogenase from Lactococcus lactis ${ }^{32}$. The resulting L-lactate CRISPRi library was characterized by NGS and contained 10494 unique sgRNA clones. To screen the library for a secreted product, we used droplet encapsulation and microfluidics sorting ${ }^{33,34}$. First, the L-lactate CRISPRi library was grown in shake-flasks, and gene repression was induced by addition of aTc. Cell aliquots were taken after $36 \mathrm{~h}$ and $66 \mathrm{~h}$ to assay for L-lactate productivity (Methods, Supplementary Fig. 10). The productivity assay involved encapsulation of cells in droplets, followed by a picoinjection of each droplet with the components of a fluorescent L-lactate assay. In each droplet, secreted L-lactate reacts with the assay, allowing droplets to be sorted based on fluorescence. The cultivation and two-time point productivity assays were done on two separate occasions, resulting in four sorting runs. For each run, approximately 180,000 cell-containing droplets were screened and 36,000 droplets were sorted. Cells could not be reliably recovered on agar plates after sorting, so we performed PCR of the sgRNA region directly from sorted droplets, followed by NGS library preparation and quantification. In each sorted sample, 1500-5000 unique clones were detected with confidence ( $>32$ reads; Methods) (Supplementary Data 4 ). Due to the small number of sorted cells and high variability between replicates, it was not possible to determine the significance of enrichment for each clone in the sorted populations. To assess which clones produced more L-lactate than average, we used instead the criteria that a clone had to be at least 3-times more abundant in the sorted fraction relative to the unsorted fraction. This criterium returned between 500 to 900 clones per sorted sample. Further filtering of clones enriched in at least two of the four sorted samples returned 397 clones, 266 harbored sgRNAs targeting ORFs and 131 harbored sgRNAs targeted to ncRNAs. Twenty-three clones were enriched in three of the four samples, and one clone was enriched in all four samples. 

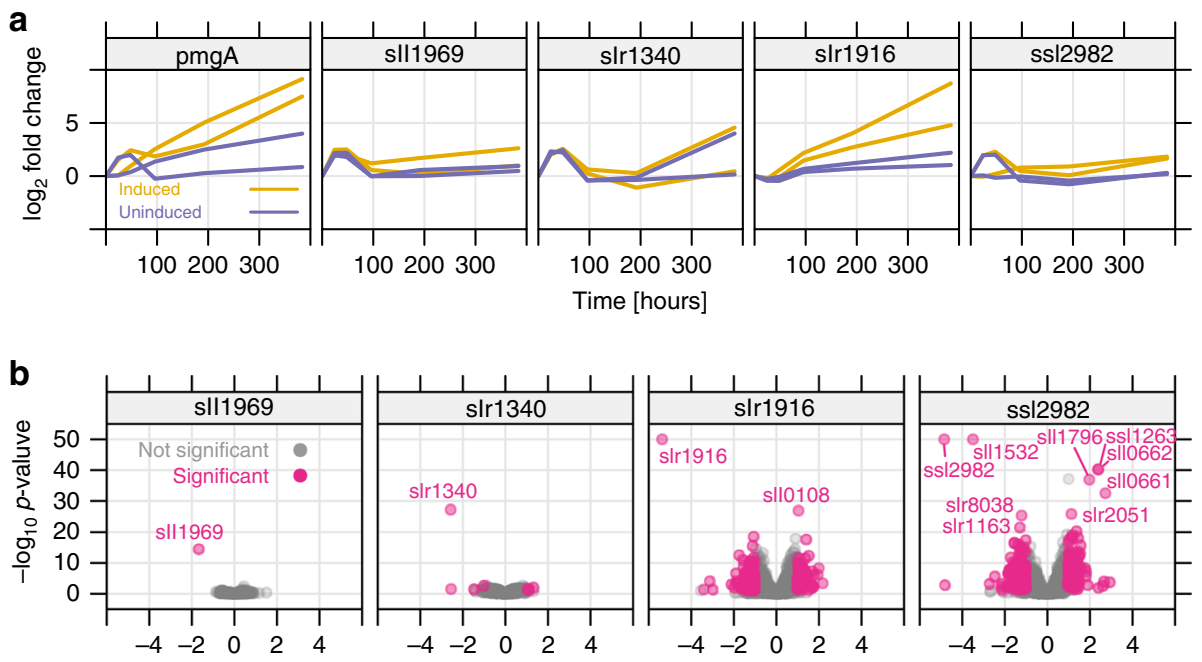

$\log _{2}$ fold change

C

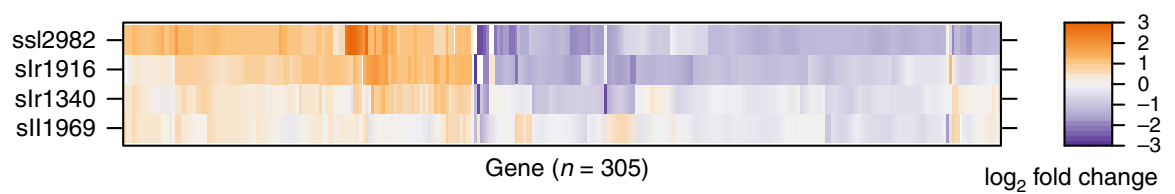

d

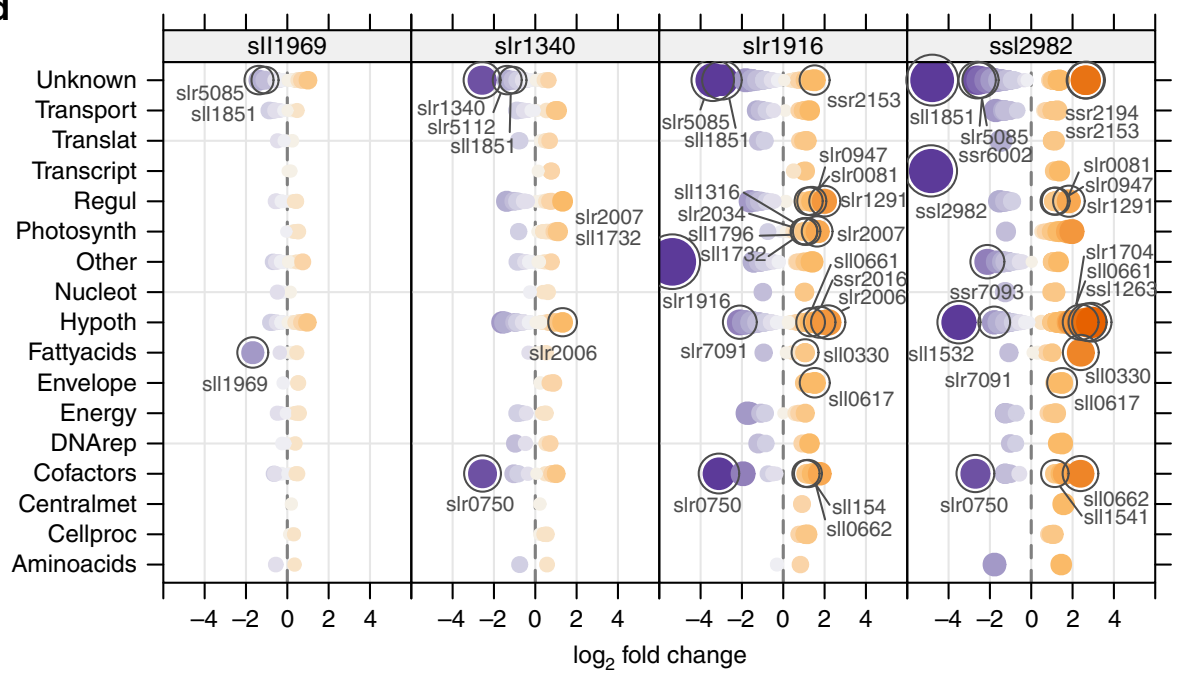

Fig. 4 Transcriptomics of faster-growing mutants. a Enrichment of faster-growing sgRNA mutants in library competition (turbidostat) experiment at L300 (300 $\mu \mathrm{mol}$ photons $\left.\mathrm{m}^{-2} \mathrm{~s}^{-1}\right), \log _{2}$ fold-change of read count over time ( $\left.n=2 \mathrm{sgRNAs}\right)$. $\mathbf{b}$ Volcano plot representation of transcriptomics data from four reconstructed sgRNA mutants. Shows $\log _{2}$ fold-change for gene expression compared to the control strain (sgRNA-NTO), against adjusted $p$-value for each gene. Gray-non-significantly different genes, pink-significantly different genes (threshold: negative $\log _{10} p$-value $\geq 2$; absolute $\log _{2}$ fold-change $\geq 1$ ). The $p$-value for three sgRNA mutants was outside the plotting region and was restricted to $-\log _{10}$ of 50 for visibility. c Heat map representation of transcriptomics data from four reconstructed sgRNA mutants. Shows all 305 genes that were significantly different in at least one of the four mutants, clustered into two different groups based on similarity of gene expression. d Significantly different genes are sorted by Cyanobase pathways. Genes of particular interest were highlighted (see text for details). Blue and orange indicates negative and positive $\log _{2}$ fold-change, respectively. Size of symbols increases with increasing absolute change. Source data underlying Fig. 4b, $d$ are provided as a Source Data file.

Though nearly half of the enriched clones targeted genes of unknown function, it is possible to derive engineering strategies from the annotated targets (Table 1). Alterations in carbon flux in cyanobacteria can be achieved by restriction of nutrient uptake or assimilation ${ }^{35-37}$. Enriched targets in nutrient uptake include glutamate dehydrogenase $(g d h A)$, glutamate synthase $(g l t B)$, and glutamine synthase $\left(g \ln A\right.$ ), repression of these would restrict $\mathrm{NH}_{3}$ assimilation, as well as a nitrogen transporter ( $n r t D 2)$ and a phosphorous transporter $(p s t C)$. Direct alteration of carbon flux was also apparent among enriched clones. For example, repression of 3-phosphoglycerate dehydrogenase (serA), citrate synthase ( $g l t A)$, and phosphoketolase (slr0453) could each be expected to increase pools of pyruvate, the precursor to Llactate ${ }^{31,38,39}$. A second engineering strategy is suggested by target genes in electron transport and energy metabolism. Computational ${ }^{40,41}$ and experimental ${ }^{42,43}$ studies have shown that efficient production of some biochemicals would require lowering the ATP/NADPH ratio in the cyanobacteria cell. The 
a

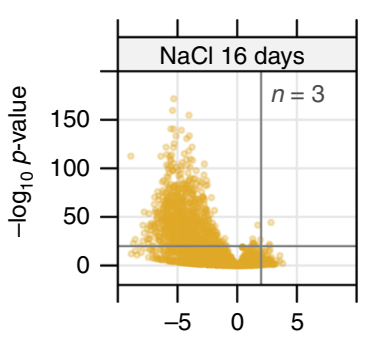

Induced

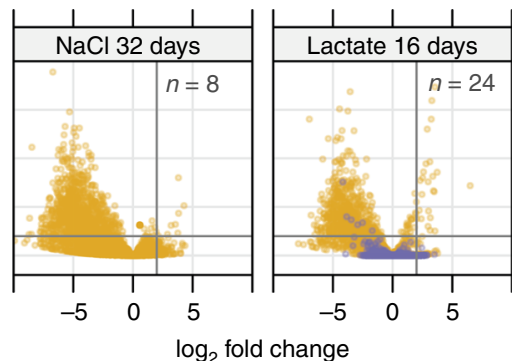

$\log _{2}$ fold change

b

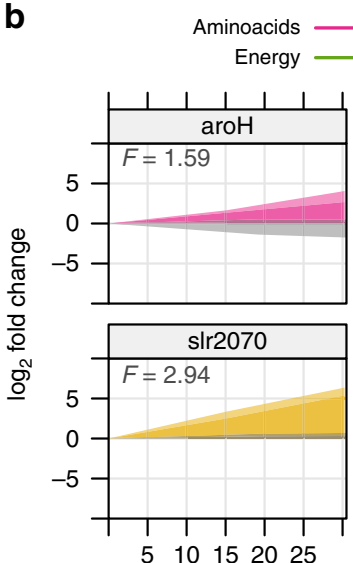

$$
\text { Energy }
$$
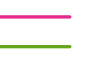

Hypoth Regul

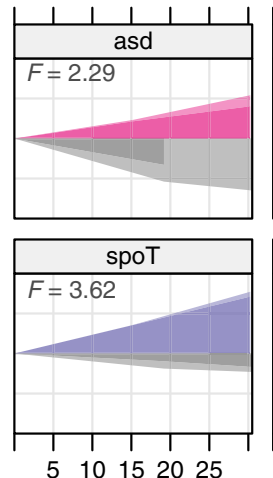

Generations

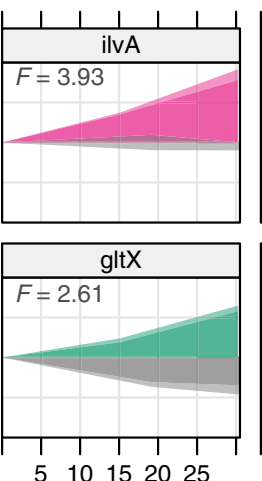

$5 \quad 10152025$
Uninduced

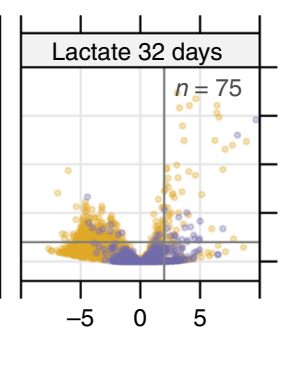

Translat
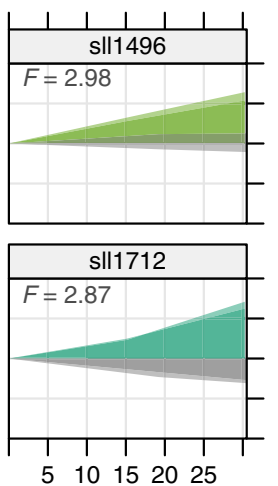

c

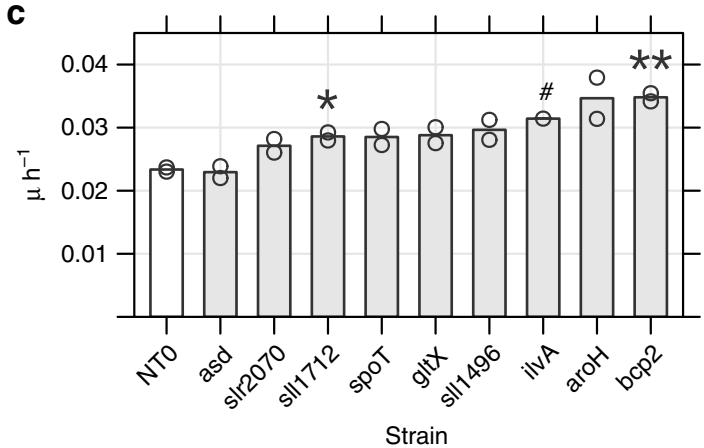

d

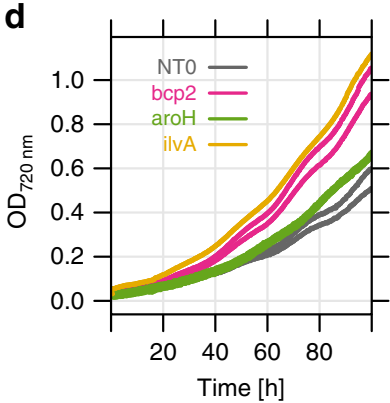

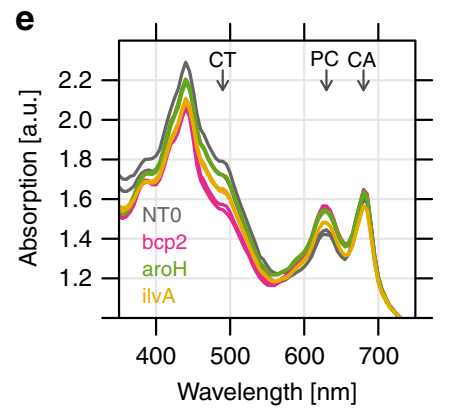

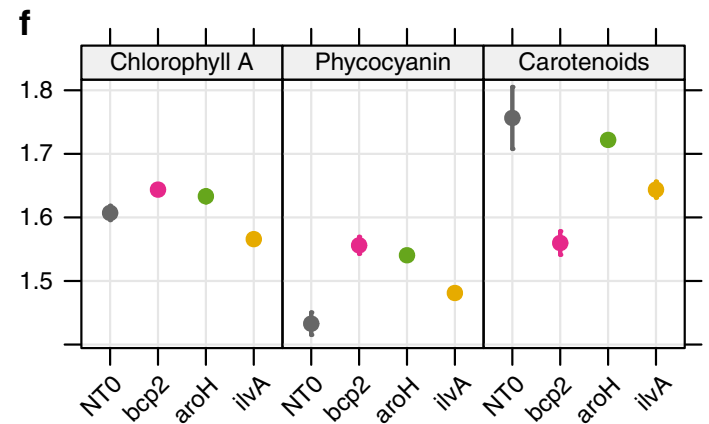

enriched targets ssr2016 and ndhD2 are both involved in ATPgenerating cyclic electron flow around PSI in Synechocystis ${ }^{44}$. $S d h B$ is a subunit of succinate dehydrogenase contributing to respiration. The flavodiiron protein flv3 (sll0550) catalyzes photoreduction of $\mathrm{O}_{2}$ during stress conditions. Knockout of $f l v 3$ was shown to lower cellular ATP/NADPH ratio in Synechocys$t_{i s^{43}}$. Finally, the CCA-tRNA nucleotidyltransferase $p c n B$, essential for tRNA maturation and thus protein synthesis, was also enriched.
We reconstructed eight clones for validation of L-lactate productivity, representing targets within nutrient uptake, carbon flux, and redox and energy generation (Table 1). In a first screen, we cultivated clones in shake-flasks. Cultures were induced for gene repression 2 days prior to inoculation. Only the gltA and $p c n B$ strains had a significantly higher L-lactate titer than the control strain (Fig. 6a). The effect was enhanced when titers were normalized to cell density, indicating a redirection of carbon flux from biomass formation to product ${ }^{38}$. Two of the potentially 
Fig. 5 SgRNA clones with improved L-lactate tolerance. a Volcano plot showing enrichment of 75 sgRNAs during cultivation with added $0.1 \mathrm{M}$ sodium Llactate after $32 \mathrm{~d}$, (threshold: $\log _{2}$ fold-change $\geq 2,-\log _{10} p$-value $\geq 20$ ). Only 8 sgRNAs were enriched in a $0.1 \mathrm{M} \mathrm{NaCl}$ control cultivation. b Eight genes where both sgRNAs were enriched above average in $0.1 \mathrm{M} \mathrm{L-lactate}$ cultivation (coloured) but not $\mathrm{NaCl}$ cultivation (gray). Color indicates association to a cyanobase pathway. $F$ - mean fitness score of two sgRNAs. c Mean growth rate $\mu\left(h^{-1}\right)$ of selected, reconstructed knockdown strains $(n=2$ independent replicate cultivations) over the first $80 \mathrm{~h}$ of batch cultivation with L-lactate added to $0.1 \mathrm{M}$. Symbols show significance between the control strain (NTO) and mutants using 1-sided student's $t$-test. ${ }^{*} p$-value $\leq 0.05,{ }^{* *} p$-value $\leq 0.01$ (sll1712, $p=0.019 ;$ bcp2, $p=0.006$ ), \#-only 1 replicate was used for ilvA mutant. d Example of growth advantage in batch culture with L-lactate added to $0.1 \mathrm{M}$ of sgRNA clones bcp2, aroH, and ilvA. e Absorption spectra of fastest-growing sgRNA mutants and NTO in $0.1 \mathrm{M} \mathrm{L-lactate.} \mathrm{Arrows} \mathrm{mark} \mathrm{absorption} \mathrm{maxima} \mathrm{for} \mathrm{pigments.} \mathrm{CT} \mathrm{carotenoids,} \mathrm{PC} \mathrm{phycocyanin,} \mathrm{CA}$ chlorophyll A. f Relative pigment absorption for carotenoids, phycocyanin, and chlorophyll A for the selected strains with L-lactate added to $0.1 \mathrm{M}$. Source data underlying Fig. 5a, b are provided as a Source Data file.

Table 1 Selection of sgRNA clones enriched by droplet sorting.

\section{sgRNA}

gdhA_25*

ssr2016_62*

gltA_41*

pntB_19

pcnB_64*

serA_10*

gltB_3*

ndhD2_15

sdhB_26*

Entry85_13

pstC_41

slr0453_10
Appearances

4

3

3

3

3

2

2

2

2

2

2

2
Effect on growth

None

Impaired

Impaired

None

Impaired

Impaired

Impaired

None

None

None

None

None
Gene function

Glutamate dehydrogenase

Ferredoxin:plastoquinone reductase, cyclic electron flow

Citrate synthase

Transhydrogenase subunit

Nucleotidyltransferase

Phosphoglycerate dehydrogenase

Glutamate synthase

$\mathrm{NDH}-1$ complex subunit, cyclic electron flow

Succinate dehydrogenase

atpA (ATP synthase) asRNA

Phosphate transporter

Phosphoketolase

Note: Mutants were identified from a CRISPRi library based on L-lactate productivity. Appearance is the number of sorting runs (out of four) where that clone had an enrichment factor of at least 2 (normalized abundance in sorted population/normalized abundance in unsorted population). Clones where growth was impaired are denoted. These clones were significantly depleted in the L300 dataset after four generations (ssr2016_62, padj $=1.6 \mathrm{e}^{-94} ;$ gltA_ $41, p_{\text {adj }}=3.1 \mathrm{e}^{-53} ;$ pcnB_64, $p_{\text {adj }}=1.5 \mathrm{e}^{-58} ;$ serA_10, padj $=3.6 \mathrm{e}^{-21} ;$ gltB_3, padj $=0.015 ;$ multiple-hypothesis adjusted $p$-values calculated by DESeq2). An asterisk denotes sgRNA mutants selected for reconstruction and validation. Source data are provided as a Source Data file.

redox-altered mutants $(s d h B, s s r 2016)$ were tested in a photonfluxostat reactor, where light intensity was gradually increased with cell density to ensure a fixed light dosage per cell ${ }^{45}$. This cultivation mode was expected to activate alternative electron flow reactions for an extended time, so as to amplify any effects of repressing these on L-lactate productivity. In photonfluxostat mode, the $s d h B$ and $s s r 2016$ clones again had higher L-lactate titers than the control strain, but variability was high and the effect was not statistically significant (Fig. 6b). We note that titers and specific productivities were lower for all strains in the photonfluxostat mode than in shake-flasks, which could be due to altered gas transfer or different perceived light intensities.

\section{Discussion}

We have primarily used the CRISPRi repression library to identify potential mutants with improved industrial phenotypes, which we then validated with screening of individual clones. By providing fitness contributions of all genes in a certain condition, the CRISPRi library reveals patterns in how cyanobacteria could solve certain physiological challenges. There were multiple genetic avenues for enhancing the growth rate of Synechocystis, though they converged on a similar transcriptome that upregulates photoprotection and electron-transport around PSI. An increase in PSI activity at high light can dissipate pressure in the electron-transport chain; a similar phenomenon was reported to contribute to high-light tolerance and faster growth in Synechococcus elongatus UTEX 2973 compared to its close relative Synechococcus PCC $7942^{46}$. This is also in-line with a recent modeling study, which showed that the rate of ATP and NADPH generation exerted the most control over carbon fixation rates in Synechocystis ${ }^{47}$. Interestingly, four out of five sgRNA targets in top faster-growing strains are potentially regulator proteins
( $p m g A, s l r 1916, s s l 2982, s l r 1340)$. This finding is reminiscent of the mutation in the master regulator RpaA of Synechococcus elongatus UTEX 2973 that drastically alters the transcriptome and is necessary for unlocking fast growth ${ }^{48}$. The RNAP omega subunit (ssl2982) is critical for acclimation of Synechocystis from low to high $\mathrm{CO}_{2}{ }^{49}$. That repression of ssl2982 provides a growth advantage at high light and high $\mathrm{CO}_{2}$ is surprising. However, our experimental setup differs from that used previously, in that we perform a knockdown of ssl2982, and that cells are already preacclimated to high $\mathrm{CO}_{2}$ before repression.

Care should be taken when using results from CRISPRi libraries to determine gene essentiality. We used 2 sgRNAs targeting each ORF; recent studies have shown that more are needed to ensure statistical significance when assessing gene essentiality $^{12}$. Furthermore, a confident determination of gene essentiality from CRISPRi data would require consideration of a gene's place in an operon. Data from an E. coli CRISPRi library showed a strong polar effect, where targeting the first gene in an operon also represses downstream genes, while the reverse polar effect, i.e., repression of downstream genes affecting expression of upstream genes, was not prominent ${ }^{50}$. For Synechocystis, transcription start sites have been mapped in detail for several growth conditions and are annotated in the reference genome (NC_000911.1 $)^{15}$. Therefore, fitness data for individual genes can be considered with their operon context. For example, the pyk2 gene (sll1275) encoding pyruvate kinase 2 is at the $5^{\prime}$ end of an operon that also contains sll1276 (probable iron transporter) and $r e c F$ downstream. In our data, both sgRNA clones targeting pyk2 showed severe growth defects, suggesting it is important for cell growth. However, one sgRNA clone targeting sll1276 also shows a strong growth defect. Considering previous attempts to knockout sll1276 were unsuccessful ${ }^{51}$, it is likely that sll1276 is essential for 
$\mathbf{a}$

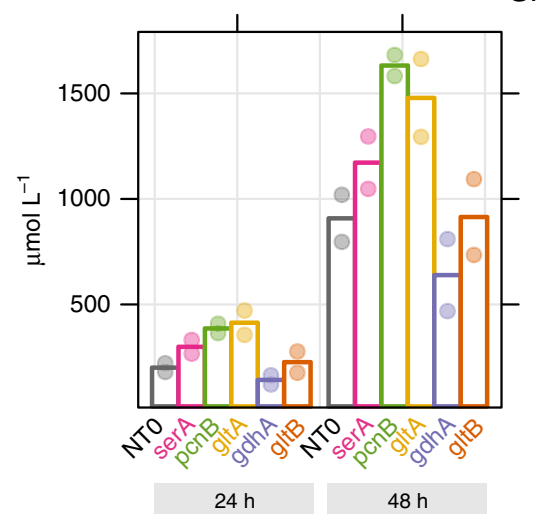

Shake flask

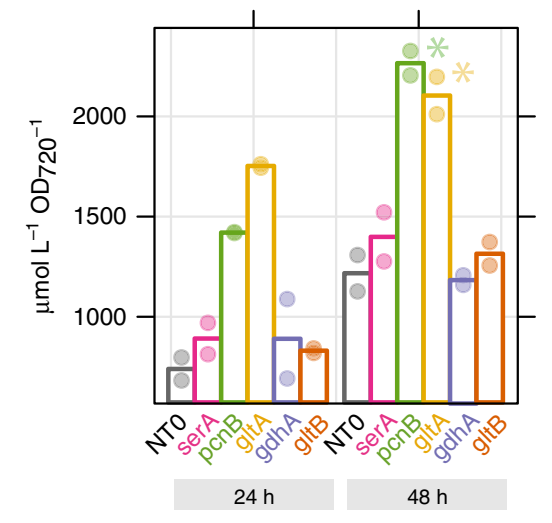

b

'Photonfluxostat'
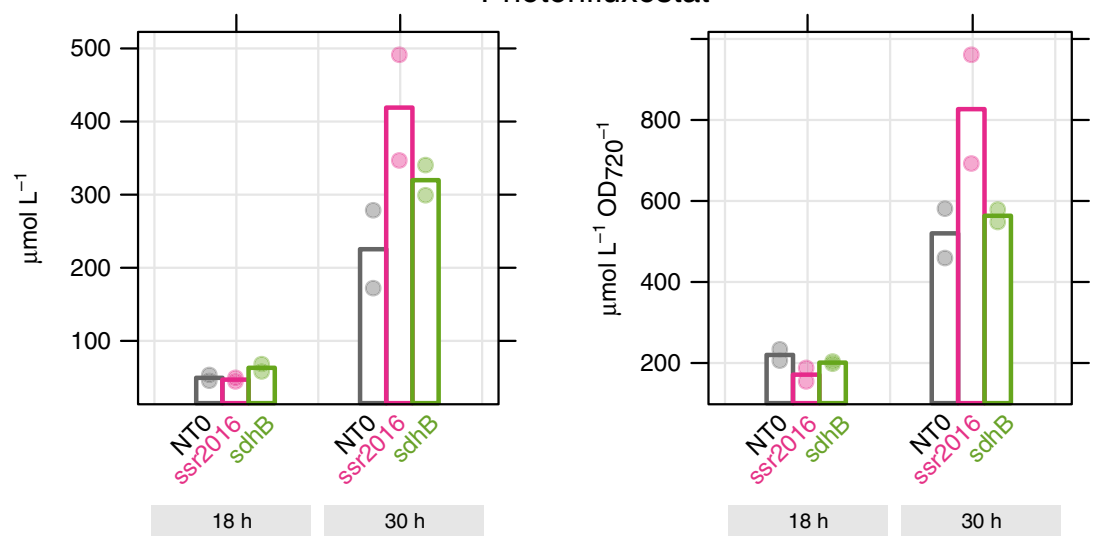

Fig. 6 SgRNA clones with increased L-lactate production. Mutants with potentially increased L-lactate productivity were discovered using a fluorescenceactivated droplet sorting assay. Seven selected mutants were reconstructed, cultivated, and product titer determined in $\mu \mathrm{mol}$ per liter, and $\mu \mathrm{mol}$ per liter per biomass $\left(O D_{720} \mathrm{~nm}\right)$. a Productivity of selected mutants cultivated in axenic shake-flask cultures $(\mathrm{n}=2$, each strain assayed in two independent replicates). L-lactate concentration was measured after 24 and 48 h. NTO - control sgRNA with no target site in Synechocystis genome. *-significant with $p \leq 0.05$, two-sided student's $t$-test, $p c n B, p=0.016$, gltA, $p=0.021$. Bars are average values and individual values are shown as points. $\mathbf{b} \mathrm{A}$ subset of two

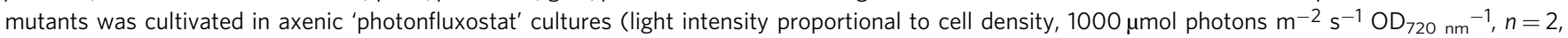
each strain assayed in 2 separate experiments). L-lactate concentration was measured after 18 and 30 h. Bars are average values and individual values are shown as points. Source data are provided as a Source Data file.

cell growth. Based on the sgRNA data alone, we cannot unambiguously conclude that pyk2 is essential for growth, as the downstream sll1276 is also likely repressed in those clones.

For $\sim 50 \%$ of the genes with low fitness scores in our data, one sgRNA was significantly depleted from the library and the other was not (Supplementary Fig. 11). There are several lines of evidence which suggest that this discrepancy is due to weak binding of one of the sgRNAs (a false-negative) instead of off-target binding of an sgRNA to an essential gene elsewhere (false-positive). First, while repression efficiency of sgRNAs targeting near the start codon is typically $>90 \%$, it can be as low as $50 \%$, so partial repression of an essential gene may not elicit a phenotype $^{13}$. Second, $86 \%(283 / 329)$ of genes predicted to be essential by Flux Balance Analysis of a Synechocystis genome-scale model had at least one associated sgRNA clone significantly depleted ${ }^{52}$ $\left(\mathrm{p}_{\text {adj }}<0.005\right.$; Supplementary Data 5$)$. There is also a $75 \%$ agreement in our calculated fitness scores for genes in central carbon metabolism to their orthologs in Synechococcus PCC 7942, the latter determined by Tn-Seq ${ }^{8}$ (Supplementary Data 6). Third, offtarget binding by dCas 9 was shown to be problematic for strongly expressed dCas $9^{50}$, while our genome-integrated $d \operatorname{Cas} 9$ was driven by a weak promoter. However, dCas 9 is potent, as we observed gene repression for some targets even in the absence of the inducer (Cluster 1 in Fig. 2). Finely-graded dCas9 expression may require native, metal-sensitive promoters ${ }^{53}$, or addition of translational-level control such as riboswitches ${ }^{54}$.

The CRISPRi library has a unique advantage over gene knockouts for engineering bioproduction in that the level of essential genes can be titrated, allowing perturbation of the core metabolic network ${ }^{55}$. Cultures where a 'metabolic switch' shifts metabolism away from growth can be more productive ${ }^{14,38,56,57}$. By coupling the CRISPRi library to a fluorescence assay, we were able to screen thousands of potential knockdowns for increased productivity, giving a test of many metabolic engineering strategies previously proposed by computational modeling. However, several limitations were apparent in the library-droplet microfluidics workflow. CRISPRi induction must be optimally timed before sorting. The $4 \mathrm{~h}$ incubation period where cells secrete L-lactate generates high singlecell variability and is dependent on cell shading, though we attempted to counter this with a high screening depth. This short incubation period was necessary to prevent saturation of the fluorescence assay. Larger droplet sizes would allow for longer incubation times. In batch validation, not all of the enriched clones from the droplet sorting gave higher L-lactate titer and there were apparent tradeoffs between productivity and carbon partitioning in some clones. Therefore, we propose that the CRISPRi library is most useful for revealing principles for guiding engineering, and that clones must be validated. 
It is easy to envision expanding an inducible CRISPRi library to other screens. Strains with faster growth rate may not be robust under stress conditions. For example, the pmgA and slr1916 mutants grew faster than the control strain in L100, L300, and LD conditions, but slower under lactate stress. Further, $p m g A$ and slr1916 knockout strains are known to be glucose sensitive in the light, as photosystem stoichiometry cannot adapt to additional reductant supply from sugar metabolism ${ }^{21,22}$. Repression of ssl2982 increases growth in L300 condition, but a knockout mutant is known to be sensitive to shifts in $\mathrm{CO}_{2}$ and temperature $^{58}$. Challenging the CRISPRi library to fluctuating conditions would allow a deeper study of the tradeoff between growth speed and robustness. Since the two fastest-growing mutants found here were also glucose sensitive, it could be worthwhile to add a screen for photomixotrophic growth, which results in flux through alternative glycolytic pathways ${ }^{59}$. Small transposon libraries $(300$ mutants) have previously been used to identify genes required for phototaxis by screening for colony smearing, ${ }^{60}$ a CRISPRi library could accelerate identification of genes involved in motility. A fluorescence-activated cell sorting (FACS) screen for cell fluorescence could be useful in mapping the photobleaching program during nutrient limitation and screens for recovery ability from starvation could identify carbon metabolism pathways involved in resuscitation ${ }^{52}$. Finally, libraries limited to a subset of target genes would be amenable to multiplexing of gene repression ${ }^{61}$.

\section{Methods}

Genetic constructs and cloning of sgRNA library. A catalytically dead Cas9 (dCas9, mutations D10A and H840A) from Streptococcus pyogenes was used for gene repression in this study and the Synechocystis base strain containing the

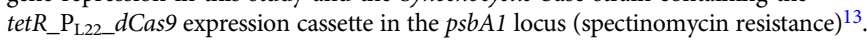
The sgRNA library oligos were synthesized on a $12 \mathrm{~K}$ chip by CustomArray Inc., USA (see Supplementary Data 1 for a list of all sgRNA sequences). Plasmid pMD19$\mathrm{T}$ (Takara) was used to create the sgRNA library. First, the homology region around locus slr0397, the PL22_sgRNA-NT0 expression cassette, and a kanamycin resistance gene were cloned into pMD19-T by BioBrick assembly. The sgRNA library oligos were then cloned into this vector using Golden Gate assembly. NEB 10-beta Competent $E$. coli cells (New England BioLabs) were used for transformation and $\sim 1,200,000 \mathrm{E}$. coli colonies were obtained. All colonies were collected, resuspended in $\mathrm{LB}$, pooled together, and then cultivated overnight in LB. The plasmid DNA was extracted using the ThermoFisher Maxi plasmid extraction kit. Natural transformation was used to transform the plasmid $(10 \mu \mathrm{g})$ in Synechocystis ${ }^{62}$, where 300,000 colonies were obtained. Colonies were collected, resuspended in fresh BG-11 and pooled. The pooled Synechocystis library was stored at $-80^{\circ} \mathrm{C}$. To re-create specific sgRNAs for clone validation, we used overlap-extension PCR of a template sgRNA to introduce the protospacer ${ }^{13}$. All primers are listed in Supplementary Data 7. The Llactate-secreting Synechocystis strain was created by cloning the ldh gene with L39R substitution from Lactococcus lactis under the Ptrc promoter ${ }^{32}$. This gene construct was inserted into the genome at locus slr0168 with a chloramphenicol resistance cassette. This strain was subsequently transformed with the tetR_P $\mathrm{P}_{\mathrm{L22} \_} d \operatorname{Cas} 9$ cassette and sgRNA library as described above, resulting in a L-lactate-producing Synechocystis sgRNA library. All strains and plasmids are available from the authors upon request.

sgRNA library design. Two sgRNAs were designed for each open reading frame (ORF) and non-coding RNA in the Synechocystis genome (Reference genome NCBI NC_000911.1, [https://www.ncbi.nlm.nih.gov/nuccore/NC_000911.1]). ORF sequence annotations were obtained from NCBI (downloaded on 18.03.2016). Locations of non-coding RNAs were from Kopf and Hess ${ }^{15,63}$. An in-house Python script was used to create protospacer sequences as close to the transcription start site (TSS) or translation start codon (ATG) as possible, within the following criteria: Target regions were required to be within $500 \mathrm{bp}$ from the known TSS or within $75 \%$ of total gene length, absence of $\mathrm{G}_{6}$ and $\mathrm{T}_{4}$, and GC content between 25 and $75 \%$. Target sequences were searched according to the pattern $5^{\prime}-\mathrm{CCN}[20-25$ bases]T-3'. The $5^{\prime}-\mathrm{CCN}$ ensured a $5^{\prime}-\mathrm{NGG}-3^{\prime}$ PAM site on the coding strand, and $3^{\prime} \mathrm{T}$ was to ensure binding of the $5^{\prime}$ end of the sgRNA, known to have an A when transcribed from promoter $\mathrm{P}_{\mathrm{L} 22}$ in Synechocystis ${ }^{64}$. For each target fitting these criteria, potential off-target regions in the genome were then identified. An offtarget binding site defined as having fewer than two mismatches in the PAMproximal $17 \mathrm{bp}$ region of the proposed sgRNA. Both NGG and NAG PAMs and both strands were considered. Then all sgRNA candidates for a gene, the two sgRNAs with the least off-targets were selected. If possible, sgRNAs were selected that were at least $10 \mathrm{bp}$ apart from each other. The in-house Python script is publicly available at [https://github.com/KiyanShabestary/2019_CRISPRi_library].
Cultivation in photobioreactor turbidostats. The Synechocystis sgRNA library was cultivated in an 8-tube photobioreactor (Multi-Cultivator MC-1000-OD, Photon System Instruments, Drasov, CZ). The system was customized to perform turbidostat cultivation ${ }^{16}$. Reactors $(65 \mathrm{~mL})$ were bubbled with $1 \% \mathrm{v} / \mathrm{v} \mathrm{CO}$ in air $(2.5 \mathrm{~mL} / \mathrm{min})$ at $30^{\circ} \mathrm{C}$, and light intensity was controlled by a computer program. The $\mathrm{OD}_{720 \mathrm{~nm}}$ and $\mathrm{OD}_{680 \mathrm{~nm}}$ were measured every $15 \mathrm{~min}$. The turbidity set point was $\mathrm{OD}_{720 \mathrm{~nm}}=0.2$ and $22 \mathrm{~mL}$ fresh BG-11 was added to dilute the culture once the set point was exceeded. Antibiotics $(25 \mu \mathrm{g} / \mathrm{mL}$ spectinomycin, $25 \mu \mathrm{g} / \mathrm{mL}$ kanamycin) and anhydrotetracycline inducer $(500 \mathrm{ng} / \mathrm{mL})$ was added to the culture and the reserve BG-11 media used for dilution. For LD cultures, the light regime (12 h light $-12 \mathrm{~h}$ dark) followed a sinusoidal function with maximum light intensity at $300 \mu \mathrm{mol} \mathrm{m}{ }^{-2} \mathrm{~s}^{-1}(\mathrm{~L}(\mathrm{t})=300 * \sin (\pi / 43200 * \mathrm{t})$ where $\mathrm{t}=$ cultivation time in seconds). For turbidostat cultivations with added L-lactate, light intensity was $100 \mu \mathrm{mol} \mathrm{m}^{-2} \mathrm{~s}^{-1}$ and either $100 \mathrm{mM}$ sodium L-lactate or $100 \mathrm{mM} \mathrm{NaCl}$ was added to the BG-11 before inoculation and medium $\mathrm{pH}$ was adjusted to 7.8. All turbidostat cultivations described in this work were performed as four independent replicates. To sample for NGS, $15 \mathrm{~mL}$ of culture volume was harvested by centrifugation $\left(5,000 \times g\right.$ for $\left.5 \mathrm{~min}, 25^{\circ} \mathrm{C}\right)$. Cells were collected and stored at $-20^{\circ} \mathrm{C}$ Batch cultivations for growth rate or L-lactate quantification of selected sgRNA mutants were performed in the same conditions as turbidostat cultures, but with light at $300 \mu \mathrm{mol}$ photons $\mathrm{m}^{-2} \mathrm{~s}^{-1}$. Mutants were pre-cultivated in BG-11 with antibiotics $(25 \mu \mathrm{g} / \mathrm{mL}$ spectinomycin, $25 \mu \mathrm{g} / \mathrm{mL}$ kanamycin) and aTc inducer (added to $500 \mathrm{ng} / \mathrm{mL}$ ) in a climatic chamber (Percival Climatics SE-1100 with 100 $\mu \mathrm{E} / \mathrm{s} / \mathrm{m}^{2}$ illumination, $1 \% \mathrm{v} / \mathrm{v} \mathrm{CO}_{2}$ at $30^{\circ} \mathrm{C}$ ) for 3 days, and then inoculated into the photobioreactor to $\mathrm{OD}_{730 \mathrm{~nm}}=0.05$ before growth measurements began.

Next-generation sequencing of sgRNA region. A two-step PCR procedure was carried out for NGS library preparation using sgRNA library plasmid from E.coli or genomic DNA from Synechocystis library as template. Genomic DNA was extracted from Synechocystis cell pellets $\left(15 \mathrm{~mL}\right.$ culture at $\left.\mathrm{OD}_{720 \mathrm{~nm}} 0.2\right)$ using GeneJET Genomic DNA Purification Kit (Thermo Fisher Scientific). 1st step PCR was performed using the primer pair LUYA593/LUYA594, which amplify sgRNAs from the plasmid or genome and add adaptors for NGS. The PCR product was analyzed on Agilent 2100 Bioanalyzer (Agilent Technologies) and gel purified using GeneJET Gel Extraction Kit (Thermo Fisher Scientific). The purified DNA was used as template to perform the 2nd step PCR using NEBNext Multiplex Oligos for Illumina (Dual Index Primers Set 1) (New England Biolabs), followed by Bioanalyzer analysis and gel purification. Purified DNA was quantified using Qubit fluorometer 2.0 (Thermo Fisher Scientific) and then pooled. The NGS was performed on Illumina NextSeq 500 system using NextSeq 500/550 High Output v2 kit (75 cycles). In a typical NGS run, 40 samples were analyzed simultaneously, providing 50-100 reads per sgRNA per sample (Supplementary Fig. 12). We used sickle 1.33 [https://github.com/najoshi/sickle] to trim (75 nt) and clean reads. A custom python script was used to assign and count reads to each sgRNA, available at [https://github.com/KiyanShabestary/2019_CRISPRi_library].

Fitness score calculation. The gradual, sgRNA-mediated depletion of clones from the library allows estimation of the contribution to cellular fitness for each individual gene. Here, we defined the fitness $F$ of a mutant as the area under the curve (AUC) for $\log _{2}$ fold-change sgRNA abundance $\left(\log _{2}\right.$ FC) at a number of generations $\left(\mathrm{n}_{\text {gen }}\right)$ since induction, normalized by maximum generations.

$$
\begin{gathered}
F=\frac{\operatorname{AUC}\left(\mathrm{n}_{\text {gen }}, \log _{2} \mathrm{FC}\right)}{\operatorname{maximum}\left(\mathrm{n}_{\text {gen }}\right)} \\
\Delta F=F_{\mathrm{L} 300}-F_{\mathrm{L} 100}
\end{gathered}
$$

Differential fitness $\Delta F$ between two conditions, e.g., L300 and L100, was calculated according to Eqs. 1 and 2.

RNA extraction and sequencing. Synechocystis fast growing mutant strains as well as the control strain (sgRNA-NT0) were cultivated in photobioreactor in turbidostat mode under the same conditions as they were enriched (see above). At 3 days post induction, $40 \mathrm{~mL}$ culture $\left(\mathrm{OD}_{730 \mathrm{~nm}}=0.2\right)$ was sampled. Cells were collected by centrifugation for $5 \mathrm{~min}$ at $4{ }^{\circ} \mathrm{C}$, and total RNA was extracted immediately afterwards. Ribosomal rRNA was depleted using Illumina Ribo-Zero rRNA Removal Kit (Bacteria). Library preparation was carried out using NEBNext Ultra II Directional RNA Library Prep Kit (New England Biolabs) following manufacturers guidelines. Libraries were sequenced on Illumina NextSeq 500 System using NextSeq 500/550 High Output v2 kit (75 cycles). RNA sequencing reads were filtered and mapped to the genome using Ribopipe ${ }^{65}$. Filtering entailed adapter removal with cutadapt 1.18 , quality trimming with sickle 1.33 , and rRNA and tRNA removal using Bowtie 1.2.2 and reference sequences from the Synechocystis genome (Reference genome NCBI NC_000911.1, [https://www.ncbi.nlm. nih.gov/nuccore/NC_000911.1]). Bowtie v. 1.2.2 was used to map the filtered reads to the Synechocystis genome. The number of reads mapping to each gene in each sample was counted and formatted using custom Python and R scripts available at [https://github.com/Asplund-Samuelsson/ribopipe]. RNA sequencing data were initially processed as described before for NGS sequencing of library data. DESeq2 was used to determine fold changes between conditions as well as significance 
(three independent biological replicates). Significant genes were selected based on the following two criteria: absolute $\log _{2} \mathrm{FC} \geq 1$, adjusted $p$-value $\leq 0.05$ ). Unsupervised clustering of significantly different genes based on expression in all mutants was performed as described for NGS data analysis.

Statistical analysis of library competition data. All analyses were performed using the $\mathrm{R}$ programming language and are documented in $\mathrm{R}$ markdown notebooks available at [https://m-jahn.github.io/]. Data for all competition experiments performed with the sgRNA library can be accessed at [https://m-jahn.shinyapps.io/ ShinyLib/]. First, data tables from different sequencing runs were merged into a single master table. For simplicity, harvesting time-points 12 and 30 days for the sodium chloride condition (NACL) were re-labelled as 16 and 32 days to correspond to time-points of all other samples. This did not influence the calculation of generation time or fitness score, and was done only to display these samples along with corresponding L-lactate samples. The R package DESeq2 was used to determine fold changes between conditions as well as significance metrics (multiple hypothesis adjusted $p$-value, Benjamini-Hochberg procedure ${ }^{66}$. Fold changes and $p$-values were determined using four independent biological replicates for all cultivations. Gene-wise annotation was added based on Uniprot (IDs, protein properties, GO terms) and CyanoBase (functional categories). Altogether 7119 unique sgRNAs corresponding to 3541 unique genes (without non-coding RNAs) were included in the analysis. The coverage per sample in terms of quantified sgRNAs and median read count per gene is shown in Supplementary Fig. 12.

Unsupervised clustering of sgRNA data. To cluster sgRNAs based on depletion/ enrichment pattern, a dissimilarity matrix was computed using R's dist function with distance measure euclidean. Clustering was performed using function hclust with method ward.D2. Silhouette analysis was performed to find the optimal number of clusters (silhouetteAnalysis from package silhouette) and showed equally good separation for 3-9 clusters. A number of six clusters was chosen representing sgRNAs with decreasing level of depletion (1-4) as well as two clusters with unchanged sgRNAs ( 5 and 6 ). These two clusters were separated by a group of sgRNAs (around $25 \%$ of all) that showed a spurious, temporary enrichment for two specific time-points ( 1 and 2 day measurements). This temporary enrichment was most likely due to technical variation (e.g., in sample preparation) and has no biological explanation. Clusters 5 and 6 were therefore combined in cluster 5 (Fig. 2a).

Gene-ontology enrichment. For gene-ontology (GO) term enrichment, the TopGO package by Alexa et al. was used to determine GO terms associated with sgRNAs for clusters 1-4 (TopGO method 'Fisher, eliminating' ${ }^{67}$. The resulting list of GO terms was filtered by dispensability scores obtained using REVIGO (http:// revigo.irb.hr/, threshold $\leq 0.5$ ). Furthermore, GO terms annotated with less than 5 or more than 200 unique sgRNA/genes or $p$-value $>0.03$ were filtered out.

Enrichment of sgRNA mutants with increased L-lactate tolerance. To find genes involved in L-lactate tolerance, sgRNAs enriched specifically for presence of sodium L-lactate but not sodium chloride were selected (threshold: $\log _{2} \mathrm{FC} \geq 2$, $-\log _{10} p$-value $\geq 20$ ). Twenty-four and 75 sgRNAs were found to be enriched after 16 and 32 days, respectively (Fig. $5 \mathrm{a}$ ). The 24 sgRNAs enriched after 16 days were included in the set of 75 sgRNAs enriched after 32 days. In contrast to the 75 sgRNAs that were enriched, thousands of sgRNAs were depleted during growth in the presence of L-lactate. To find sgRNAs specifically depleted under L-lactate but not sodium chloride, a differential fitness score was calculated between the two conditions $\left(d F=F_{\mathrm{NaCl}}-F_{L a c}\right)$. The top 200 sgRNAs with highest $d F$ were selected and six genes were found with both sgRNAs strongly depleted (see also Supplementary Fig. 9).

Estimation of mutant growth rates. The change in a mutant's abundance within the total population, combined with the population average growth rate (known from the dilution rate of the turbidostat), allows estimation of the growth rate of each mutant. The rate of enrichment or depletion of a mutant $\left(\mu_{\text {diff }}\right)$ was defined as the population growth rate $\left(\mu_{p o p}\right)$ subtracted by the mutant's individual growth rate $\left(\mu_{m u t}\right)$ (Eq. 3)

$$
\mu_{\text {diff }}=\mu_{\text {pop }}-\mu_{\text {mut }}
$$

The depletion of a mutant from the library can be modeled as a function of time, where the mutant fraction $f$ at time point $t$ becomes:

$$
f(t)=f(t=0) \times\left(1-\left(\mu_{\text {pop }}-\mu_{\text {mut }}\right)\right)^{t}
$$

The mutant fraction $f$ at different times and the average growth rate of the population are known parameters, allowing to estimate growth rate for all mutants (Eq. 4).

Correlation between gene expression variability and fitness. To correlate gene fitness (obtained from sgRNA library competition experiments) with variability in gene expression, the proteomics dataset PRIDE PXD009582 was used ${ }^{16}$. The dataset contains mass spectrometry based protein measurements of Synechocystis for 2000 proteins at five different growth rates, each for $\mathrm{CO}_{2}$ and light limitation. The variability of a protein was defined as growth-rate dependent change in abundance, and determined as $p$-value from analysis of variance (ANOVA). If a protein changed abundance significantly with growth rate (either up or downregulation) a lower $p$-value was obtained. Proteins were binned into groups according to $p$-value ranges $(1-0.1,0.1-0.05,0.05-0.01,0.01-0.001,<0.001)$ and fitness score of sgRNAs associated with the respective proteins in a group were plotted.

Absorption spectrum of L-lactate-tolerant mutants. Selected mutants as well as the control strain (sgRNA-NT0) were cultivated in $30 \mathrm{~mL}$ BG-11 supplemented with $100 \mathrm{mM}$ sodium L-lactate in shaking flasks at $100 \mu \mathrm{mol}$ photons $\mathrm{m}^{-2} \mathrm{~s}^{-1}$ continuous light, and $500 \mathrm{ng} / \mathrm{mL}$ aTc was added at the beginning of the cultivation. $0.5 \mathrm{~mL}$ cell culture was sampled after induction for 3 days and cells were collected by centrifugation at $5000 \times g$ for $10 \mathrm{~min}$. Cells were then washed twice with $1 \mathrm{~mL}$ PBS by centrifugation and resuspension, and then resuspended in $400 \mu \mathrm{L}$ PBS. Two times $100 \mu \mathrm{L}$ resuspended cells were transferred to a transparent 96-well plate. Absorption spectra of cell samples were obtained in the range of 350-800 nm using a photospectrometer (SpectraMax M5, Molecular Devices). Absorption spectra were normalized to the reference $A_{720} \mathrm{~nm}$ and relative chlorophyll a, phycocyanin

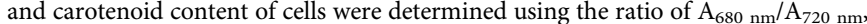
$\mathrm{A}_{630 \mathrm{~nm}} / \mathrm{A}_{720 \mathrm{~nm}}$, and $\mathrm{A}_{490 \mathrm{~nm}} / \mathrm{A}_{720 \mathrm{~nm}}$, respectively.

Droplet microfluidic screening of L-lactate-producing sgRNA library. The L-lactate-producing Synechocystis sgRNA library was grown in shake-flasks in a climatic chamber. Cultures were supplemented with antibiotics $(12.5 \mu \mathrm{g} / \mathrm{mL}$ chloramphenicol, $25 \mu \mathrm{g} / \mathrm{mL}$ spectinomycin) and aTc $(1 \mu \mathrm{g} / \mathrm{mL})$. For the productivity assay, cells were harvested $\left(\mathrm{OD}_{720 \mathrm{~nm}}=0.4-0.6\right)$, washed to $\mathrm{OD}_{720 \mathrm{~nm}}=$ 0.15 , and encapsulated into droplets ${ }^{68}$. A mix of HFE-7500 oil and $1 \%(w / w)$ EA surfactant droplet stabilizer (RainDance Technologies) was loaded into a Gastight 5 -mL glass syringe (Hamilton). Cells were encapsulated in $10 \mathrm{pL}$ droplets using a flow rate of $400 \mu \mathrm{L} / \mathrm{h}$ for the aqueous solution and $2000 \mu \mathrm{L} / \mathrm{h}$ for the oil. The emulsion was collected in a 1-mL plastic syringe at a withdrawal flow rate of 2000 $\mu \mathrm{L} / \mathrm{h}$. The syringes were connected to the chip by polyether ether ketone tubing, and flow rates were controlled by neMESYS syringe pumps (Cetoni GmbH). The droplet emulsion was incubated in an illuminated syringe (approximately $150 \mu \mathrm{E} / \mathrm{s}$ / $\mathrm{m}^{2}$ ) for $4 \mathrm{~h}$ for L-lactate secretion. Three $\mathrm{pL}$ of a fluorescence-based L-lactate assay mixture (Cayman Chemical) was then injected into each droplet using a picoinjection chip ${ }^{69}$. The emulsion was injected at a flow rate of $70 \mu \mathrm{L} / \mathrm{h}$, spacer oil separated the droplets at a flow rate of $500 \mu \mathrm{L} / \mathrm{h}$ and the lactate assay mixture was injected at $30 \mu \mathrm{L} / \mathrm{h}$. The picoinjected emulsion was collected in a $1 \mathrm{~mL}$ plastic syringe protected from light. After $40 \mathrm{~min}$ of picoinjection, the collected emulsion was gently mixed and re-injected into a droplet sorting device(Supplementary Fig. 9). The flow rates used were $1000 \mu \mathrm{L} / \mathrm{h}$ for the spacer oil and side oil, and the emulsion was injected at $100 \mu \mathrm{L} / \mathrm{h}$, corresponding to a droplet sorting rate of 1.5 $\mathrm{kHz}$. Sorted droplets were collected using a withdrawal rate of $1000 \mu \mathrm{L} / \mathrm{h}$. Approximately 1,800,000 droplets were screened and the top $2 \%$ most fluorescent droplets were sorted, corresponding to approximately 36,000 droplets. After sorting, the collected emulsion was broken with $10 \% \mathrm{v} / \mathrm{v} 1 \mathrm{H}, 1 \mathrm{H}, 2 \mathrm{H}, 2 \mathrm{H}$-perfluoro-1octanol (Sigma Aldrich), followed by centrifugation and evaporation to remove the residual liquid. Cells were resuspended in DMSO and heated at $95^{\circ} \mathrm{C}$ for $2 \mathrm{~min}$ to release genomic content. The cell lysate was used as template to directly amplify the sgRNA region using primer pair LUYA271/LUYA300. Then adaptors were incorporated into the PCR product using primer pair LUYA593/LUYA594, followed by NGS library preparation as described above.

Droplet microfluidics NGS data filtering. Using NGS obtained on each sorted droplet fraction, we could map 5 million reads per sample, covering to $8000-10,000$ unique sgRNAs. sgRNAs with fewer than 32 mapped reads in a sample were removed from that sample. To calculate 'enrichment factors' for each sgRNA, the relative abundance of the sgRNA in the sorted droplet was divided by its relative abundance in the total library before droplet encapsulation. Next, sgRNAs where the enrichment factor differed $\geq 10$-fold between replicates were removed. The remaining sgRNAs were then ranked by enrichment factor for each sorted sample sgRNAs at least three times more abundant in sorted fraction relative to unsorted fraction were given a score of 1 for that sample. Clones with a score $2 / 4$, summed over all samples were determined to have potential to increase L-lactate productivity.

L-lactate production in batch and photonfluxostat. The L-lactate-producing Synechocystis sgRNA library mutants were first cultivated in shake-flasks in a climatic chamber. Cultures were pre-cultivated in BG-11 supplemented with antibiotics ( $12.5 \mu \mathrm{g} / \mathrm{mL}$ chloramphenicol, $25 \mu \mathrm{g} / \mathrm{mL}$ kanamycin) and induced with aTc $(1 \mu \mathrm{g} / \mathrm{mL}) 2$ days prior to the start of the experiment. Then mutants were transferred to shake-flasks or in an 8-tube photobioreactor (see library cultivation methods) with a starting $\mathrm{OD}_{720 \mathrm{~nm}}$ of 0.1 , supplemented with aTc and antibiotics as stated above. Cultures in the photobioreactor were grown in a 'photonfluxostat' mode, where light is increased based on cellular density to extend log phase. The amount of light given to the culture is equal to the cellular density multiplied by a 
light regime factor $\left(1000 \mu \mathrm{mol}\right.$ photons $\left.\mathrm{m}^{-2} \mathrm{~s}^{-1} \mathrm{OD}_{720 \mathrm{~nm}^{-1}}\right)$. L-lactate was measured using a L-lactate fluorescent kit (Cayman Chemical) according to the manufacturer's instructions.

Reporting summary. Further information on research design is available in the Nature Research Reporting Summary linked to this article.

\section{Data availability}

A reporting summary for this Article is available as a Supplementary Information file. Data supporting the findings of this work are available within the paper and its Supplementary Information files. The datasets generated and analyzed during the current study are available from the corresponding author upon request. The data for competition experiments performed with the sgRNA library can also be accessed through an interactive web application [https://m-jahn.shinyapps.io/ShinyLib/]. All sequencing data, including the RNA sequencing data described in Fig. 4, are available at the European Nucleotide Archive with accession number PRJEB35238. Proteomics data used in Supplementary Fig. 1 was from PRIDE PXD009582. The source data underlying Figs. 2, 3, 4b-d, 5a, b, and 6, Table 1, as well as Supplementary Figs 1-7, 9, and 11 are provided as a Source Data file.

\section{Code availability}

All analyses of sgRNA library competition data were performed using the $\mathrm{R}$ programming language and are documented in R markdown notebooks [https://m-jahn. github.io/]. The code used to process RNA-Seq data from raw files (read trimming, mapping to genome, PCA analysis) is deposited at GitHub [https://github.com/AsplundSamuelsson/ribopipe]. The code used to design sgRNA sequences for each gene in Synechocystis, the script to assign NGS reads to sgRNAs, and the pipeline for data filtering doplet NGS data is also available at GitHub [https://github.com/ KiyanShabestary/2019_CRISPRi_library].

Received: 30 October 2019; Accepted: 13 March 2020;

Published online: 03 April 2020

\section{References}

1. Mullineaux, C. W. Electron transport and light-harvesting switches in cyanobacteria. Front. Plant Sci. 5, 7 (2014).

2. Orf, I. et al. Can cyanobacteria serve as a model of plant photorespiration?-a comparative meta-analysis of metabolite profiles. J. Exp. Bot. 67, 2941-2952 (2016).

3. Cohen, S. E. \& Golden, S. S. Circadian rhythms in cyanobacteria. Microbiol. Mol. Biol. Rev. 79, 373-385 (2015).

4. Oliver, N. J. et al. Cyanobacterial metabolic engineering for biofuel and chemical production. Curr. Opin. Chem. Biol. 35, 43-50 (2016).

5. Perez, A. A., Chen, Q., Hernandez, H. P., Branco Dos Santos, F. \& Hellingwerf, $\mathrm{K}$. J. On the use of oxygenic photosynthesis for the sustainable production of commodity chemicals. Physiol. Plant 166, 413-427 (2019).

6. Perin, G. \& Jones, P. R. Economic feasibility and long-term sustainability criteria on the path to enable a transition from fossil fuels to biofuels. Curr. Opin. Biotechnol. 57, 175-182 (2019).

7. Price, M. N. et al. Mutant phenotypes for thousands of bacterial genes of unknown function. Nature 557, 503-509 (2018).

8. Rubin, B. E. et al. The essential gene set of a photosynthetic organism. Proc. Natl Acad. Sci. USA 112, E6634-E6643 (2015).

9. Welkie, D. G. et al. Genome-wide fitness assessment during diurnal growth reveals an expanded role of the cyanobacterial circadian clock protein KaiA. Proc. Natl Acad. Sci. USA 115, E7174-E7183 (2018).

10. Peters, J. M. et al. A comprehensive, CRISPR-based functional analysis of essential genes in bacteria. Cell 165, 1493-1506 (2016).

11. Wang, T. et al. Pooled CRISPR interference screening enables genome-scale functional genomics study in bacteria with superior performance. Nat. Commun. 9, 2475 (2018)

12. Rousset, F. et al. Genome-wide CRISPR-dCas9 screens in E. coli identify essential genes and phage host factors. PLoS Genet. 14, e1007749 (2018).

13. Yao, L., Cengic, I., Anfelt, J. \& Hudson, E. P. Multiple gene repression in cyanobacteria using CRISPRi. ACS Synth. Biol. 5, 207-212 (2016).

14. Gordon, G. C. et al. CRISPR interference as a titratable, trans-acting regulatory tool for metabolic engineering in the cyanobacterium Synechococcus sp. strain PCC 7002. Metab. Eng. 38, 170-179 (2016).

15. Kopf, M. et al. Comparative analysis of the primary transcriptome of Synechocystis sp. PCC 6803. DNA Res. 5, 527-539 (2014).

16. Jahn, M. et al. Growth of cyanobacteria is constrained by the abundance of light and carbon assimilation proteins. Cell Rep. 25, 478-486 (2018).
17. Zavrel, T. et al. Quantitative insights into the cyanobacterial cell economy. elife 8, e42508 (2019).

18. Gopalakrishnan, S., Pakrasi, H. B. \& Maranas, C. D. Elucidation of photoautotrophic carbon flux topology in Synechocystis PCC 6803 using genome-scale carbon mapping models. Metab. Eng. 47, 190-199 (2018).

19. Hendry, J. I. et al. Genome-scale fluxome of Synechococcus elongatus UTEX 2973 using transient ${ }^{13}$ C-labeling data. Plant Physiol. 179, 761-769 (2019).

20. Kucho, K. I. et al. Global analysis of circadian expression in the cyanobacterium Synechocystis sp. strain PCC 6803. J. Bacteriol. 187, 2190-2199 (2005)

21. Hihara, Y., Sonoike, K. \& Ikeuchi, M. A novel gene, $p m g A$, specifically regulates photosystem stoichiometry in the cyanobacterium Synechocystis species PCC 6803 in response to high light. Plant Physiol. 117, 1205-1216 (1998).

22. Ozaki, H., Ikeuchi, M., Ogawa, T., Fukuzawa, H. \& Sonoike, K. Large-scale analysis of chlorophyll fluorescence kinetics in Synechocystis sp. PCC 6803: identification of the factors involved in the modulation of photosystem stoichiometry. Plant Cell Physiol. 48, 451-458 (2007).

23. Baers, L. L. et al. Proteome mapping of a cyanobacterium reveals distinct compartment organisation and cell-dispersed metabolism. Plant Physiol. 181, 1721-1738 (2019).

24. Gunnelius, L. et al. The omega subunit of the RNA polymerase core directs transcription efficiency in cyanobacteria. Nucleic Acids Res. 42, 4606-4614 (2014).

25. de Porcellinis, A. J. et al. The non-coding RNA Ncr0700/PmgR1 is required for photomixotrophic growth and the regulation of glycogen accumulation in the cyanobacterium Synechocystis sp. PCC 6803. Plant Cell Physiol. 57, 2091-2103 (2016)

26. Riediger, $\mathrm{M}$. et al. Biocomputational analyses and experimental validation identify the regulon controlled by the redox-responsive transcription factor RpaB. iScience 15, 316-331 (2019).

27. Battchikova, N., Eisenhut, M. \& Aro, E. M. Cyanobacterial NDH-1 complexes: Novel insights and remaining puzzles. Biochim. Biophys. Acta Bioenerg. 1807, 935-944 (2011).

28. Clark, R. L. et al. Light-optimized growth of cyanobacterial cultures: growth phases and productivity of biomass and secreted molecules in light-limited batch growth. Metab. Eng. 47, 230-242 (2018).

29. Zakrzewska, A. et al. Genome-wide analysis of yeast stress survival and tolerance acquisition to analyze the central trade-off between growth rate and cellular robustness. Mol. Biol. Cell 22, 4435-4446 (2011).

30. Kadowaki, T., Nishiyama, Y., Hisabori, T. \& Hihara, Y. Identification of OmpR-family response regulators interacting with thioredoxin in the cyanobacterium Synechocystis sp. PCC 6803. PLoS ONE 10, e0119107 (2015).

31. Xiong, W. et al. Phosphoketolase pathway contributes to carbon metabolism in cyanobacteria. Nat. Plants 2, 15187 (2015).

32. Angermayr, S. A. et al. Exploring metabolic engineering design principles for the photosynthetic production of lactic acid by Synechocystis sp. PCC6803. Biotechnol. Biofuels 7, 99 (2014).

33. Baret, J. C. et al. Fluorescence-activated droplet sorting (FADS): Efficient microfluidic cell sorting based on enzymatic activity. Lab Chip 9, 1850-1858 (2009).

34. Bjork, S. M. \& Joensson, H. N. Microfluidics for cell factory and bioprocess development. Curr. Opin. Biotechnol. 55, 95-102 (2019).

35. Hauf, W. et al. Metabolic changes in Synechocystis PCC6803 upon nitrogenstarvation: Excess NADPH sustains polyhydroxybutyrate accumulation. Metabolites 3, 101-118 (2013).

36. Nakajima, T., Yoshikawa, K., Toya, Y., Matsuda, F. \& Shimizu, H. Metabolic flux analysis of the Synechocystis sp. PCC 6803 DeltanrtABCD Mmutant reveals a mechanism for metabolic adaptation to nitrogen-limited conditions. Plant Cell Physiol. 58, 537-545 (2017).

37. Kato, A. et al. Identification of a cyanobacterial RND-type efflux system involved in export of free fatty acids. Plant Cell Physiol. 56, 2467-2477 (2015).

38. Shabestary, K. et al. Targeted repression of essential genes to arrest growth and increase carbon partitioning and biofuel titers in cyanobacteria. ACS Synth. Biol. 7, 1669-1675 (2018)

39. Anfelt, J. et al. Genetic and nutrient modulation of acetyl-CoA levels in Synechocystis for n-butanol production. Microb. Cell Fact. 14, 167 (2015).

40. Erdrich, P., Knoop, H., Steuer, R. \& Klamt, S. Cyanobacterial biofuels: New insights and strain design strategies revealed by computational modeling. Microb. Cell Fact. 13, 128 (2014).

41. Shabestary, K. \& Hudson, E. P. Computational metabolic engineering strategies for growth-coupled biofuel production by Synechocystis. Metab. Eng. Commun. 3, 216-226 (2016).

42. Wang, X. et al. Enhanced limonene production in cyanobacteria reveals photosynthesis limitations. Proc. Natl Acad. Sci. USA 113, 14225-14230 (2016).

43. Thiel, K. et al. Redirecting photosynthetic electron flux in the cyanobacterium Synechocystis sp. PCC 6803 by the deletion of flavodiiron protein Flv3. Microb. Cell Fact. 18, 189 (2019). 
44. Yeremenko, N. et al. Open reading frame ssr2016 is required for antimycin Asensitive photosystem I-driven cyclic electron flow in the cyanobacterium Synechocystis sp. PCC 6803. Plant Cell Physiol. 46, 1433-1436 (2005).

45. Du., W. et al. Photonfluxostat: a method for light-limited batch cultivation of cyanobacteria at different, yet constant, growth rates. Algal Res. 20, 118-125 (2016).

46. Ungerer, J., Lin, P.-C., Chen, H.-Y. \& Pakrasi, H. B. Adjustments to photosystem stoichiometry and electron transfer proteins are key to the remarkably fast growth of the cyanobacterium Synechococcus elongatus UTEX 2973. mBio 9, e02327-17 (2018).

47. Janasch, M., Asplund-Samuelsson, J., Steuer, R. \& Hudson, E. P. Kinetic modeling of the Calvin cycle identifies flux control and stable metabolomes in Synechocystis carbon fixation. J. Exp. Bot. 70, 973-983 (2019).

48. Ungerer, J., Wendt, K. E., Hendry, J. I., Maranas, C. D. \& Pakrasi, H. B. Comparative genomics reveals the molecular determinants of rapid growth of the cyanobacterium Synechococcus elongatus UTEX 2973. Proc. Natl Acad. Sci. USA 115, E11761-E11770 (2018).

49. Kurkela, J., Hakkila, K., Antal, T. \& Tyystjärvi, T. Acclimation to high CO2 requires the w subunit of the RNA polymerase in Synechocystis. Plant Physiol. 174, 172-184 (2017)

50. Cui, L. et al. A CRISPRi screen in E. coli reveals sequence-specific toxicity of dCas9. Nat. Commun. 9, 1912 (2018).

51. Katoh, H., Grossman, A. R., Hagino, N. \& Ogawa, T. A gene of Synechocystis sp. strain PCC 6803 encoding a novel iron transporter. J. Bacteriol. 182, 6524-6531 (2000).

52. Klotz, A. et al. Awakening of a dormant cyanobacterium from nitrogen chlorosis reveals a genetically determined program. Curr. Biol. 26, 2862-2872 (2016).

53. Guerrero, F., Carbonell, V., Cossu, M., Correddu, D. \& Jones, P. R. Ethylene synthesis and regulated expression of recombinant protein in Synechocystis sp. PCC 6803. PLoS ONE 7, e50470 (2012).

54. Canadas, I. C., Groothuis, D., Zygouropoulou, M., Rodrigues, R. \& Minton, N. P. RiboCas: a universal CRISPR-based editing tool for clostridium. ACS Synth. Biol. 8, 1379-1390 (2019).

55. Beuter, D. et al. Selective enrichment of slow-growing bacteria in a netabolism-wide CRISPRi library with a TIMER protein. ACS Synth. Biol. 7, 2775-2782 (2018).

56. Burg, J. M. et al. Large-scale bioprocess competitiveness: the potential of dynamic metabolic control in two-stage fermentations. Curr. Opin. Chem. Eng. 14, 121-136 (2016).

57. Klamt, S., Mahadevan, R. \& Hadicke, O. When do two-stage processes outperform one-stage processes? Biotechnol. J. 13, 1700539 (2018).

58. Gunnelius, L. et al. The $\omega$ subunit of RNA polymerase is essential for thermal acclimation of the cyanobacterium Synechocystis sp. PCC 6803. PLoS ONE 9, e112599 (2014).

59. Chen, X. et al. The Entner-Doudoroff pathway is an overlooked glycolytic route in cyanobacteria and plants. Proc. Natl Acad. Sci. USA 113, 5441-5446 (2016)

60. Bhaya, D., Takahashi, A., Shahi, P. \& Grossman, A. R. Novel motility mutants of Synechocystis strain PCC 6803 generated by in vitro transposon mutagenesis. J. Bacteriol. 183, 6140-6143 (2001).

61. Kaczmarzyk, D., Cengic, I., Yao, L. \& Hudson, E. P. Diversion of the longchain acyl-ACP pool in Synechocystis to fatty alcohols through CRISPRi repression of the essential phosphate acyltransferase PlsX. Metab. Eng. 45, 59-66 (2018).

62. Cheah, Y. E., Albers, S. C. \& Peebles, C. A. A novel counter-selection method for markerless genetic modification in Synechocystis sp. PCC 6803. Biotechnol. Prog. 29, 23-30 (2013).

63. Kopf, M. \& Hess, W. R. Regulatory RNAs in photosynthetic cyanobacteria. FEMS Microbiol. Rev. 39, 301-315 (2015).

64. Huang, H. H. \& Lindblad, P. Wide-dynamic-range promoters engineered for cyanobacteria. J. Biol. Eng. 7, 10 (2013).

65. Karlsen, J., Asplund-Samuelsson, J., Thomas, Q., Jahn, M. \& Hudson, E. P. Ribosome profiling of Synechocystis reveals altered ribosome allocation at carbon starvation. mSystems 3, e00126-18 (2018).
66. Love, M. I., Huber, W. \& Anders, S. Moderated estimation of fold change and dispersion for RNA-seq data with DESeq2. Genome Biol. 15, 550 (2014).

67. Alexa, A., Rahnenfuhrer, J. \& Lengauer, T. Improved scoring of functional groups from gene expression data by decorrelating GO graph structure. Bioinformatics 22, 1600-1607 (2006).

68. Hammar, P. et al. Single cell screening of photosynthetic growth and lactate production by cyanobacteria. Biotechnol. Biofuels 8, 193 (2015).

69. Abate, A. R., Hung, T., Mary, P., Agresti, J. J. \& Weitz, D. A. High-throughput injection with microfluidics using picoinjectors. Proc. Natl Acad. Sci. USA 107, 19163-19166 (2010).

\section{Acknowledgements}

We thank Filipe Brancos dos Santos, Aniek van der Woude and Klaas Hellingwerf for helpful discussions. This work was funded by the Swedish Research Council [201606160], the Swedish Foundation for Strategic Research SSF [RB14-0013], ÅForsk Foundation [16-611], and the European Union's Horizon 2020 research and innovation program under Grant Agreement No. 760994 (ENGICOIN project). Open access funding provided by Royal Institute of Technology.

\section{Author contributions}

K.S. and L.Y. designed and cloned the CRISPRi library, performed competition experiments and next-generation sequencing. L.Y. cloned and cultivated growth mutants. K.S. cloned and cultivated lactate over-producing mutants. K.S., S.B., and H.J. performed droplet sorting. J.A.S. performed RNA-Seq data processing. M.J. performed data processing and analysis for competition experiments and RNA-Seq. All authors designed the experiments. L.Y., K.S., M.J., and E.P.H. wrote the manuscript. All authors read and approved the manuscript.

\section{Competing interests}

The authors declare no competing interests.

\section{Additional information}

Supplementary information is available for this paper at https://doi.org/10.1038/s41467020-15491-7.

Correspondence and requests for materials should be addressed to E.P.H.

Peer review information Nature Communications thanks Patrik Jones, and the other, anonymous, reviewer(s) for their contribution to the peer review of this work. Peer reviewer reports are available.

Reprints and permission information is available at http://www.nature.com/reprints

Publisher's note Springer Nature remains neutral with regard to jurisdictional claims in published maps and institutional affiliations.

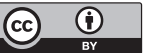

Open Access This article is licensed under a Creative Commons Attribution 4.0 International License, which permits use, sharing, adaptation, distribution and reproduction in any medium or format, as long as you give appropriate credit to the original author(s) and the source, provide a link to the Creative Commons license, and indicate if changes were made. The images or other third party material in this article are included in the article's Creative Commons license, unless indicated otherwise in a credit line to the material. If material is not included in the article's Creative Commons license and your intended use is not permitted by statutory regulation or exceeds the permitted use, you will need to obtain permission directly from the copyright holder. To view a copy of this license, visit http://creativecommons.org/ licenses/by/4.0/.

(C) The Author(s) 2020 